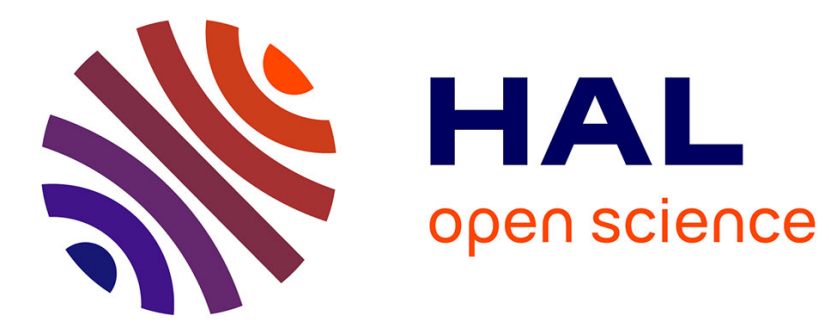

\title{
Prime 3-uniform hypergraphs
}

Pierre Ille, Abderrahim Boussaïri, Brahim Chergui, Mohamed Zaidi

\section{To cite this version:}

Pierre Ille, Abderrahim Boussaïr, Brahim Chergui, Mohamed Zaidi. Prime 3-uniform hypergraphs.

Graphs and Combinatorics, 2021, 37, pp.2737-2760. hal-03362671

\section{HAL Id: hal-03362671 \\ https://hal.science/hal-03362671}

Submitted on 1 Oct 2021

HAL is a multi-disciplinary open access archive for the deposit and dissemination of scientific research documents, whether they are published or not. The documents may come from teaching and research institutions in France or abroad, or from public or private research centers.
L'archive ouverte pluridisciplinaire HAL, est destinée au dépôt et à la diffusion de documents scientifiques de niveau recherche, publiés ou non, émanant des établissements d'enseignement et de recherche français ou étrangers, des laboratoires publics ou privés. 


\title{
Prime 3-uniform hypergraphs
}

\author{
Abderrahim Boussaïri*§ Brahim Chergui $* \ddagger \quad$ Pierre Illet \\ Mohamed Zaidi*\|
}

July 29, 2021

\begin{abstract}
Given a 3-uniform hypergraph $H$, a subset $M$ of $V(H)$ is a module of $H$ if for each $e \in E(H)$ such that $e \cap M \neq \varnothing$ and $e \backslash M \neq \varnothing$, there exists $m \in M$ such that $e \cap M=\{m\}$ and for every $n \in M$, we have $(e \backslash\{m\}) \cup\{n\} \in E(H)$. For example, $\varnothing, V(H)$ and $\{v\}$, where $v \in V(H)$, are modules of $H$, called trivial. A 3-uniform hypergraph is prime if all its modules are trivial. Given a prime 3-uniform hypergraph, we study its prime, 3-uniform and induced subhypergraphs. Our main result is: given a prime 3-uniform hypergraph $H$, with $|V(H)| \geq 4$, there exist $v, w \in V(H)$ such that $H-\{v, w\}$ is prime.
\end{abstract}

Mathematics Subject Classifications (2010): 05C65, 05C20.

Key words: hypergraph, 3-uniform, module, prime.

\section{Introduction}

Let $H$ be a 3-uniform hypergraph. A tournament $T$, with the same vertex set as $H$, is a realization of $H$ if the edges of $H$ are exactly the 3-element subsets of the vertex set of $T$ that induce 3 -cycles. In [3], we characterized the 3 -uniform hypergraphs that admit realizations (see [4, Problem 1]). To obtain our characterization, we introduced a new notion of a module for hypergraphs. By using the modular decomposition tree, we demonstrated that a 3-uniform hypergraph is realizable if and only if all its prime, 3-uniform and induced subhypergraphs are realizable (see [3, Theorem 13]). Moreover, given a realizable 3-uniform hypergraph $H$, we proved that $H$ is prime if and only if its realizations are prime (see [3, Theorem 12]). These results lead us to study

\footnotetext{
*Faculté des Sciences Aïn Chock, Département de Mathématiques et Informatique, $\mathrm{Km} 8$ route d'El Jadida, BP 5366 Maarif, Casablanca, Maroc

${ }^{\dagger}$ Aix Marseille Univ, CNRS, I2M, Marseille, France

$\S$ aboussairi@hotmail.com

${ }^{\ddagger}$ cherguibrahim@gmail.com

I pierre.ille@univ-amu.fr

$\|_{\text {zaidi.fsac@gmail.com }}$
} 
the prime, 3-uniform and induced subhypergraphs of a prime 3-uniform hypergraph. Precisely, consider a prime 3-uniform hypergraph $H$. We are interested in the integers $n \in\{3, \ldots,|V(H)|-1\}$ for which there exists $W \subseteq V(H)$ satisfying $|W|=n$ and $H[W]$ is prime. We adopt the same layout as that followed for prime tournaments. The main results on prime tournaments are recalled in Section 2. Similar results were obtained for prime digraphs [10], for prime binary relational structures [12], or for prime 2-structures [7].

At present, we formalize our presentation. We consider only finite structures. A hypergraph $H$ is defined by a vertex set $V(H)$ and an edge set $E(H)$, where $E(H) \subseteq 2^{V(H)} \backslash\{\varnothing\}$. In the sequel, we consider only hypergraphs $H$ such that

$$
E(H) \subseteq 2^{V(H)} \backslash(\{\varnothing\} \cup\{\{v\}: v \in V(H)\}) .
$$

Given $k \geq 2$, a hypergraph $H$ is $k$-uniform if

$$
E(H) \subseteq\left(\begin{array}{c}
V(H) \\
k
\end{array}\right)
$$

Let $H$ be a hypergraph. With each $W \subseteq V(H)$, we associate the subhypergraph $H[W]$ of $H$ induced by $W$, which is defined on $V(H[W])=W$ by $E(H[W])=$ $\{e \in E(H): e \subseteq W\}$.

A realization of a 3-uniform hypergraph is defined as follows. To begin, we associate with each tournament a 3-uniform hypergraph in the following way.

Definition 1. The 3-cycle is the tournament $C_{3}=(\{0,1,2\},\{01,12,20\})$. Given a tournament $T$, the $C_{3}$-structure of $T$ is the 3-uniform hypergraph $C_{3}(T)$ defined on $V\left(C_{3}(T)\right)=V(T)$ by

$$
E\left(C_{3}(T)\right)=\left\{X \subseteq V(T): T[X] \text { is isomorphic to } C_{3}\right\} \text { (see [4]). }
$$

Definition 2. Given a 3-uniform hypergraph $H$, a tournament $T$, with $V(T)=$ $V(H)$, realizes $H$ if $H=C_{3}(T)$. We say also that $T$ is a realization of $H$. Lastly, we say that a 3 -uniform hypergraph is realizable if it admits a realization.

In [3], we characterized the realizable 3-uniform hypergraphs. We used mainly the following notion of a module.

Definition 3. Let $H$ be a hypergraph. A subset $M$ of $V(H)$ is a module of $H$ if for each $e \in E(H)$ such that $e \cap M \neq \varnothing$ and $e \backslash M \neq \varnothing$, there exists $m \in M$ such that $e \cap M=\{m\}$, and for every $n \in M$, we have

$$
(e \backslash\{m\}) \cup\{n\} \in E(H) .
$$

Given distinct $v, w \in V(H)$, we say that $v$ and $w$ are twins $[2,5]$ of $H$ if $\{v, w\}$ is a module of $H$.

Definition 4. Let $S$ be a set. A family $\mathscr{F}$ of subsets of $S$ is a partitive family [6, Definition 6] on $S$ if it satisfies the following assertions.

- $\varnothing \in \mathscr{F}, S \in \mathscr{F}$, and for every $x \in S,\{x\} \in \mathscr{F}$. 
- For any $M, N \in \mathscr{F}, M \cap N \in \mathscr{F}$.

- For any $M, N \in \mathscr{F}$, if $M \cap N \neq \varnothing, M \backslash N \neq \varnothing$ and $N \backslash M \neq \varnothing$, then $M \cup N \in \mathscr{F}$ and $(M \backslash N) \cup(N \backslash M) \in \mathscr{F}$.

In [3, Proposition 3], we established the following result.

Proposition 5. The family of the modules of a hypergraph is a partitive family.

As mentioned at the beginning of the section, Definition 3 is not the classic definition of a module of a hypergraph. The classic definition follows.

Definition 6. Let $H$ be a hypergraph. A subset $M$ of $V(H)$ is a module [1, Definition 2.4] of $H$ if for any $e, f \subseteq V(H)$ such that $|e|=|f|, e \backslash M=f \backslash M$, and $e \backslash M \neq \varnothing$, we have $e \in E(H)$ if and only if $f \in E(H)$.

Remark 7. Definitions 3 and 6 coincide for 2-uniform hypergraphs, that is, for graphs. They do not in the general case. Given a hypergraph $H$, a module of $H$ in the sense of Definition 3 is a module in the sense of Definition 6. The converse is not true. For instance, consider the 3-uniform hypergraph $H$ defined on $V(H)=\{0,1,2,3\}$ by $E(H)=\{013,123\}$. Clearly, $\{0,1\}$ and $\{1,2\}$ are modules of $H$ in the sense of Definition 6, but not in the sense of Definition 3. Moreover, $\{0,1,2\}$ is not a module of $H$ in the sense of Definition 6 because $013 \in E(H)$ and $023 \notin E(H)$. Consequently, Proposition 5 does not hold for the family of the modules in the sense of Definition 6.

In [3], we generalized the notion of a partitive family as follows.

Definition 8. Let $S$ be a set. A modular covering of $S$ is a function $\mathfrak{M}$ which associates with each $W \subseteq S$ a set $\mathfrak{M}(W)$ of subsets of $W$, and which satisfies the following assertions.

(A1) For each $W \subseteq S, \mathfrak{M}(W)$ is a partitive family on $W$.

(A2) For any $W, W^{\prime} \subseteq S$, if $W \subseteq W^{\prime}$, then

$$
\left\{M^{\prime} \cap W: M^{\prime} \in \mathfrak{M}\left(W^{\prime}\right)\right\} \subseteq \mathfrak{M}(W) .
$$

(A3) For any $W, W^{\prime} \subseteq S$, if $W \subseteq W^{\prime}$ and $W \in \mathfrak{M}\left(W^{\prime}\right)$, then

$$
\left\{M^{\prime} \in \mathfrak{M}\left(W^{\prime}\right): M^{\prime} \subseteq W\right\}=\mathfrak{M}(W) .
$$

(A4) Let $W, W^{\prime} \subseteq S$ such that $W \subseteq W^{\prime}$. For any $M \in \mathfrak{M}(W)$ and $M^{\prime} \in \mathfrak{M}\left(W^{\prime}\right)$, if $M \cap M^{\prime}=\varnothing$ and $M^{\prime} \cap W \neq \varnothing$, then $M \in \mathfrak{M}\left(W \cup M^{\prime}\right)$.

(A5) Let $W, W^{\prime} \subseteq S$ such that $W \subseteq W^{\prime}$. For any $M \in \mathfrak{M}(W)$ and $M^{\prime} \in \mathfrak{M}\left(W^{\prime}\right)$, if $M \cap M^{\prime} \neq \varnothing$, then $M \cup M^{\prime} \in \mathfrak{M}\left(W \cup M^{\prime}\right)$.

In [3, Proposition 5], we proved the following result. 
Proposition 9. Given a hypergraph $H$, the function defined on $2^{V(H)}$, which maps each $W \subseteq V(H)$ to the family of the modules of $H[W]$, is a modular covering of $V(H)$.

The main notion follows.

Definition 10. Let $H$ be a hypergraph. By Proposition 5, $\varnothing, V(H)$ and $\{v\}$, where $v \in V(H)$, are modules of $H$, called trivial modules. A hypergraph $H$ is indecomposable if all its modules are trivial, otherwise it is decomposable. A hypergraph $H$ is prime if it is indecomposable, with $|V(H)| \geq 3$.

Remark 11. Recall that a hypergraph $H$ is connected if for distinct $v, w \epsilon$ $V(H)$, there exists a sequence $\left(e_{0}, \ldots, e_{n}\right)$ of edges of $H$, where $n \geq 0$, satisfying $v \in e_{0}, w \in e_{n}$, and (when $\left.n \geq 1\right) e_{i} \cap e_{i+1} \neq \varnothing$ for every $0 \leq i \leq n-1$. Given a hypergraph $H$, a maximal connected subhypergraph of $H$ is called a component of $H$.

Let $H$ be a hypergraph. For each component $C$ of $H, V(C)$ and $V(H) \backslash V(C)$ are clearly modules of $H$. Therefore, a disconnected hypergraph with at least three vertices is decomposable.

\subsection{The main results}

To begin, we prove that there exist prime subhypergraphs of a small size in a prime $k$-uniform hypergraph. We prove the next lemma at the beginning of Section 3.

Lemma 12. Given a prime $k$-uniform hypergraph $H$, where $k \geq 3$, the following assertions hold

$$
\left\{\begin{array}{l}
\cup E(H)=V(H) \\
\text { and } \\
\text { for every e } \in E(H), H[e] \text { is prime. }
\end{array}\right.
$$

As for tournaments (see Notation 21), to construct prime subhypergraphs of a larger size in a prime hypergraph, we use the set $p_{(H, X)}$ defined as follows. The introduction of $p_{(H, X)}$ is classic in the study of prime structures (for instance, see [10, Definition 1] for digraphs).

Notation 13. Let $H$ be a $k$-uniform hypergraph, where $k \geq 3$. Given $X \nsubseteq V(H)$ such that $H[X]$ is prime, we consider the following subsets of $V(H) \backslash X$.

- We denote by $\operatorname{Ext}_{(H, X)}$ the set of $v \in V(H) \backslash X$ such that $H[X \cup\{v\}]$ is prime. (Hence, given $v \in V(H) \backslash X, v \in \operatorname{Ext}_{(H, X)}$ if and only if $H[X \cup\{v\}]$ is prime extension of $H[X]$.)

- We denote by $\operatorname{Dis}_{(H, X)}$ the set of $v \in V(H) \backslash X$ such that $X$ is a module of $H[X \cup\{v\}]$. (Let $v \in \operatorname{Dis}_{(H, X)}$. Since $X$ is a module of $H[X \cup\{v\}]$, we obtain $v \notin e$ for every $e \in E(H[X \cup\{v\}])$. Thus, $H[X \cup\{v\}]$ is disconnected (see Remark 11)). 
- Let $y \in X$. We denote by $\operatorname{Twi}_{(H, X)}(y)$ the set of $v \in V(H) \backslash X$ such that $\{y, v\}$ is a module of $H[X \cup\{v\}]$. (Let $y \in X$. Given $v \in V(H) \backslash X$, $v \in \operatorname{Twi}_{(H, X)}(y)$ if and only if $v$ and $y$ are twins of $H[X \cup\{v\}]$.)

The set $\left\{\operatorname{Ext}_{(H, X)}, \operatorname{Dis}_{(H, X)}\right\} \cup\left\{\operatorname{Twi}_{(H, X)}(y): y \in X\right\}$ is denoted by $p_{(H, X)}$.

The following fact constitutes the starting point of our study. It is an easy consequence of Propositions 5 and 9. We show it at the beginning of Section 3.

Fact 14. Let $H$ be a 3-uniform hypergraph. Consider $X \mp V(H)$ such that $H[X]$ is prime. The set $p_{(H, X)}$ is a partition of $V(H) \backslash X$.

The next result allows us to obtain prime subhypergraphs of a larger size in a prime 3-uniform hypergraph. We prove it in Section 4.

Theorem 15. Let $H$ be a prime 3-uniform hypergraph. Consider $X \mp V(H)$ such that $H[X]$ is prime. There exists $Y \subseteq V(H) \backslash X$ such that $1 \leq|Y| \leq 3$ and $H[X \cup Y]$ is prime.

Theorem 15 has to be compared with Corollary 24 for tournaments. The following corollary is obtained from Lemma 12 after using Theorem 15 several times.

Corollary 16. Let $H$ be a prime 3-uniform hypergraph. If $|V(H)| \geq 4$, then there exists $Y \subseteq V(H)$ such that $1 \leq|Y| \leq 3$ and $H-Y$ is prime.

We establish Corollary 16 at the end of Section 4. To improve Corollary 16, we establish the following result.

Theorem 17. Let $H$ be a 3-uniform hypergraph. Consider $X \mp V(H)$ such that $H[X]$ is prime. Set

$$
\underline{X}=\left\{y \in X: \operatorname{Twi}_{(H, X)}(y) \neq \varnothing\right\} .
$$

If $H$ is prime, then there exist $v, w \in(V(H) \backslash X) \cup \underline{X}$ such that $H-\{v, w\}$ is prime.

We prove Theorem 17 in Section 5. The next result is an easy consequence of Lemma 12 and Theorem 17. It improves Corollary 16.

Corollary 18. Let $H$ be a prime 3-uniform hypergraph. If $|V(H)| \geq 4$, then there exist $v, w \in V(H)$ such that $H-\{v, w\}$ is prime.

Corollary 18 does not hold if we require also that $v \neq w$ (see Remark 41), whereas it does for a prime tournament $T$ such that $|V(T)| \geq 7$ (see Theorem 26). In [3, Theorem 12], we proved the following result.

Theorem 19. Consider a realizable and 3-uniform hypergraph $H$. For a realization $T$ of $H$, we have $H$ is prime if and only if $T$ is prime.

It follows from Theorems 19 and 26 that the prime 3-uniform hypergraphs constructed in Remark 41 are not realizable. We conclude this section with the following problem.

Problem 20. Characterize the prime 3-uniform hypergraphs $H$ such that $H$ $\{v, w\}$ is decomposable for any distinct $v, w \in V(H)$. 


\section{Background on prime tournaments}

Let $T$ be a tournament. A subset $M$ of $V(T)$ is a module [13] of $T$ provided that for any $x, y \in M$ and $v \in V(T)$, if $x v, v y \in A(T)$, then $v \in M$. Note that the notions of a module and of a convex subset [9] coincide for tournaments. Moreover, note that the notions of a module and of an interval coincide for linear orders.

Given a tournament $T, \varnothing, V(T)$ and $\{v\}$, where $v \in V(T)$, are modules of $T$, called trivial modules. A tournament is indecomposable if all its modules are trivial, otherwise it is decomposable. A tournament $T$ is prime if it is indecomposable, with $|V(T)| \geq 3$. For instance, the 3 -cycle $C_{3}$ (see Definition 1) is prime.

Let $T$ be a strongly connected tournament (with $|V(T)| \geq 3$ ). For every $v \in V(T)$, there exists $X \subseteq V(T)$ such that $v \in X$ and $T[X]$ is isomorphic to $C_{3}$. Since $C_{3}$ is prime, we obtain

$$
v \in X,|X|=3 \text {, and } T[X] \text { is prime. }
$$

Of course, (2) holds for prime tournaments. To construct prime subtournaments of a larger size in a prime tournament, we use the set $p_{(T, X)}$ defined as follows.

Notation 21. Let $T$ be a tournament. Given $X \nsubseteq V(T)$ such that $T[X]$ is prime, consider the following subsets of $V(T) \backslash X$

- $\operatorname{Ext}_{(T, X)}$ denotes the set of $v \in V(T) \backslash X$ such that $T[X \cup\{v\}]$ is prime;

- $\operatorname{Dis}_{(T, X)}$ denotes the set of $v \in V(T) \backslash X$ such that $X$ is a module of $T[X \cup\{v\}]$

- for each $y \in X, \operatorname{Twi}_{(T, X)}(y)$ denotes the set of $v \in V(T) \backslash X$ such that $\{y, v\}$ is a module of $T[X \cup\{v\}]$.

The set $\left\{\operatorname{Ext}_{(T, X)}, \operatorname{Dis}_{(T, X)}\right\} \cup\left\{\operatorname{Twi}_{(T, X)}(y): y \in X\right\}$ is denoted by $p_{(T, X)}$.

The next lemma is basic, and its proof is easy. Close statements can be found for 2-structures in [8] (see Lemmas 5.1, 5.2, and 5.3).

Lemma 22. Given a tournament $T$, consider $X \nsubseteq V(T)$ such that $T[X]$ is prime. The set $p_{(T, X)}$ is a partition of $V(T) \backslash X$. Moreover, the following assertions hold.

1. For $v \in \operatorname{Dis}_{(T, X)}$ and $w \in V(T) \backslash\left(X \cup \operatorname{Dis}_{(T, X)}\right)$, if $T[X \cup\{v, w\}]$ is decomposable, then $X \cup\{w\}$ is a module of $T[X \cup\{v, w\}]$.

2. Given $y \in X$, for $v \in \operatorname{Twi}_{(T, X)}(y)$ and $w \in V(T) \backslash\left(X \cup \operatorname{Twi}_{(T, X)}(y)\right)$, if $T[X \cup\{v, w\}]$ is decomposable, then $\{y, v\}$ is a module of $T[X \cup\{v, w\}]$.

3. For $v, w \in \operatorname{Ext}_{(T, X)}$ such that $v \neq w$, if $T[X \cup\{v, w\}]$ is decomposable, then $\{v, w\}$ is a module of $T[X \cup\{v, w\}]$.

The next result follows from Lemma 22. 
Proposition 23. Given a prime tournament $T$, consider $X \subseteq V(T)$ such that $T[X]$ is prime. The following assertions hold.

1. If $\operatorname{Dis}_{(T, X)} \neq \varnothing$, then there exist $v \in \operatorname{Dis}_{(T, X)}$ and $w \in V(T) \backslash\left(X \cup \operatorname{Dis}_{(T, X)}\right)$ such that $T[X \cup\{v, w\}]$ is prime.

2. Given $y \in X$, if $\operatorname{Twi}_{(T, X)}(y) \neq \varnothing$, then there exist $v \in \operatorname{Twi}_{(T, X)}(y)$ and $w \in V(T) \backslash\left(X \cup \operatorname{Twi}_{(T, X)}(y)\right)$ such that $T[X \cup\{v, w\}]$ is prime.

3. If $|V(T) \backslash X| \geq 2$ and $V(T) \backslash X=\operatorname{Ext}_{(T, X)}$, then there exist $v, w \in \operatorname{Ext}_{(T, X)}$ such that $v \neq w$ and $T[X \cup\{v, w\}]$ is prime.

The next result is a simple consequence of Proposition 23.

Corollary 24 (Theorem $6.5[7]$ ). Given a prime tournament $T$, consider $X \subseteq$ $V(T)$ such that $T[X]$ is prime. If $|V(T) \backslash X| \geq 2$, then there exist $v, w \in V(T) \backslash X$ such that $v \neq w$ and $T[X \cup\{v, w\}]$ is prime.

The next result follows from (2) by applying Corollary 24 several times.

Corollary 25. For a prime tournament $T$ such that $|V(T)| \geq 5$, there exist $v, w \in V(T)$ such that $T-\{v, w\}$ is prime.

Since $v=w$ may occur in Corollary 25, it is improved as follows.

Theorem 26 (Schmerl and Trotter [12]). Given a prime tournament $T$, if $|V(T)| \geq 7$, then there exist $v, w \in V(T)$ such that $v \neq w$ and $T-\{v, w\}$ is prime.

Lastly, Theorem 26 is improved as follows.

Theorem 27 (Sayar [11]). Given a prime tournament $T$, consider $X \subseteq V(T)$ such that $T[X]$ is prime. If $|V(T) \backslash X| \geq 4$, then there exist $v, w \in V(T) \backslash X$ such that $v \neq w$ and $T-\{v, w\}$ is prime.

\section{Preliminary lemmas}

We begin the section with the following remark on Lemma 12 .

Remark 28. Consider a hypergraph $H$ such that $|V(H)| \geq 3$ and $V(H) \in E(H)$. We verify that $H$ is prime. Indeed, consider a module $M$ of $H$ such that $|M| \geq 2$. We have $|V(H) \cap M| \geq 2$. Since $V(H) \in E(H)$ and $M$ is a module of $H$, we obtain $V(H) \subseteq M$, so $M=V(H)$. Therefore, $H$ is prime.

Now, we prove Lemma 12.

Proof of Lemma 12. First, we verify that $\cup E(H)=V(H)$. Clearly, $\cup E(H)$ is a module of $H$. Since $H$ is prime, $E(H) \neq \varnothing$. Given $e \in E(H)$, we have $|e|=k$ and $e \subseteq(\cup E(H))$. Thus $|\cup E(H)| \geq k$. Since $H$ is prime, $\cup E(H)=V(H)$.

Second, let $e \in E(H)$. Since $H$ is $k$-uniform, $E(H[e])=\{e\}$. It follows from Remark 28 that $H[e]$ is prime. 
To continue, we show Fact 14.

Proof of Fact 14. First, we verify that the union of the elements of $p_{(H, X)}$ equals $V(H) \backslash X$. Let $v \in V(H) \backslash X$. If $H[X \cup\{v\}]$ is prime, then $v \in \operatorname{Ext}_{(H, X)}$. Suppose that $H[X \cup\{v\}]$ is decomposable. Consider a nontrivial module $M$ of $H[X \cup\{v\}]$. By Proposition 9 (see Assertion (A2) of Definition 8), $M \cap X$ is a module of $H[X]$. Since $H[X]$ is prime, $M \cap X$ is a trivial module of $H[X]$. Thus, we obtain $M \cap X=\varnothing, X$, or $\{y\}$, where $y \in X$. Since $M$ is a nontrivial module of $H[X \cup\{v\}]$, we have $M \cap X \neq \varnothing$. It follows that $M \cap X=X$ or $\{y\}$, where $y \in X$. We distinguish the following two cases.

- Suppose that $M \cap X=X$. Since $M$ is a nontrivial module of $H[X \cup\{v\}]$, we have $M=X$. Therefore, $v \in \operatorname{Dis}_{(H, X)}$.

- Suppose that $M \cap X=\{y\}$, where $y \in X$. Since $M$ is a nontrivial module of $H[X \cup\{v\}]$, we obtain $M=\{y, v\}$. Therefore, $v \in \operatorname{Twi}_{(H, X)}(y)$.

Second, we verify that the elements of $p_{(H, X)}$ are pairwise disjoint. Given $v \in V(H) \backslash X$, we have $v \in \operatorname{Ext}_{(H, X)}$ if and only if $H[X \cup\{v\}]$ is prime. Hence, $\operatorname{Ext}_{(H, X)} \cap \operatorname{Dis}_{(H, X)}=\varnothing$ and $\operatorname{Ext}_{(H, X)} \cap \operatorname{Twi}_{(H, X)}(y)=\varnothing$ for every $y \in X$.

Let $y \in X$. For a contradiction, suppose that there exists $v \in \operatorname{Dis}_{(H, X)} \cap$ $\operatorname{Twi}_{(H, X)}(y)$. We obtain that $X$ and $\{y, v\}$ are modules of $H[X \cup\{v\}]$. It follows from Proposition 5 that $X \backslash\{y\}$ is a module of $H[X \cup\{v\}]$. By Proposition 9 (see Assertion (A2) of Definition 8), $X \backslash\{y\}$ is a module of $H[X]$, which contradicts the fact that $H[X]$ is prime. Consequently, $\operatorname{Dis}_{(H, X)} \cap \operatorname{Twi}_{(H, X)}(y)=\varnothing$.

Finally, consider distinct $y, z \in X$. For a contradiction, suppose that there exists $v \in \operatorname{Twi}_{(H, X)}(y) \cap \operatorname{Twi}_{(H, X)}(z)$. We obtain that $\{y, v\}$ and $\{z, v\}$ are modules of $H[X \cup\{v\}]$. It follows from Proposition 5 that $\{y, z, v\}$ is a module of $H[X \cup\{v\}]$. By Proposition 9 (see Assertion (A2) of Definition 8), $\{y, z\}$ is a module of $H[X]$, which contradicts the fact that $H[X]$ is prime. Consequently,

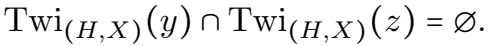

We use the following fact to prove Lemmas 31, 32, 33, and 35 .

Fact 29. Let $H$ be a 3-uniform hypergraph. Consider $X \nsubseteq V(H)$ such that $H[X]$ is prime. Let $M$ be a module of $H$. We have $M \cap X=\varnothing, M \supseteq X$ or $M \cap X=\{y\}$, where $y \in X$. Moreover, the following assertions hold.

1. If $M \cap X=\varnothing$, then all the elements of $M$ belong to the same block of $p_{(H, X)}$.

2. If $M \supseteq X$, then all the elements of $V(H) \backslash M$ belong to $\operatorname{Dis}_{(H, X)}$.

3. If $M \cap X=\{y\}$, where $y \in X$, then all the elements of $M \backslash\{y\}$ belong to $\operatorname{Twi}_{(H, X)}(y)$.

Proof. Consider a module $M$ of $H[X \cup\{v\}]$. By Proposition 9 (see Assertion (A2) of Definition 8), $M \cap X$ is a module of $H[X]$. Since $H[X]$ is prime, $M \cap X$ is a trivial module of $H[X]$. Thus, we obtain $M \cap X=\varnothing, X$, or $\{y\}$, where $y \in X$. 
For the first assertion, suppose that $M \cap X=\varnothing$. Let $v, w \in M$. By Proposition 9 (see Assertion (A2) of Definition 8), $\{v, w\}$ is a module of $H[X \cup\{v, w\}]$. It follows that the function

$$
\begin{array}{ccc}
X \cup\{v\} & \longrightarrow & X \cup\{w\} \\
v & \longmapsto & w \\
z \in X & \longmapsto & z,
\end{array}
$$

is an isomorphism from $H[X \cup\{v\}]$ onto $H[X \cup\{w\}]$. Therefore, $v$ and $w$ belong to the same block of $p_{(H, X)}$.

For the second assertion, suppose that $M \cap X=X$, so $M \supseteq X$. Let $v \in$ $V(H) \backslash M$. By Proposition 9 (see Assertion (A2) of Definition 8), $X$ is a module of $H[X \cup\{v\}]$. Therefore, $v \in \operatorname{Dis}_{(H, X)}$.

For the third assertion, suppose that $M \cap X=\{y\}$, where $y \in X$. Let $v \in M \backslash\{y\}$. By Proposition 9 (see Assertion (A2) of Definition 8), $\{v, y\}$ is a module of $H[X \cup\{v\}]$. Therefore, $v \in \operatorname{Twi}_{(H, X)}(y)$.

The remainder of the section is organized as follows. We consider a 3uniform hypergraph $H$, a subset $X$ of $V(H)$ such that $H[X]$ is prime, and distinct elements $v$ and $w$ of $V(H) \backslash X$. We examine the following cases.

- In Lemma 31 , we suppose that $v, w \in \operatorname{Dis}_{(H, X)}$.

- In Lemma 32, we suppose that $v \in \operatorname{Dis}_{(H, X)}$ and $w \in \operatorname{Twi}_{(H, X)}(y)$, where $y \in X$.

- In Lemma 33, we suppose that $v, w \in \operatorname{Twi}_{(H, X)}(y)$, where $y \in X$.

- In Lemma 35, we suppose that $v \in \operatorname{Twi}_{(H, X)}(y)$ and $w \in \operatorname{Twi}_{(H, X)}(z)$, where $y$ and $z$ are distinct elements of $X$.

In the next remark, we discuss Lemma 31 in the particular case of a 3-uniform hypergraph on 5 vertices in terms of decomposability and realizability.

Remark 30. Given a tournament $T$, consider $X \nsubseteq V(T)$ such that $T[X]$ is prime. Let $v$ and $w$ be distinct elements of $\operatorname{Dis}_{(T, X)}$. Clearly, $X$ is a module of $T[X \cup\{v, w\}]$. Hence, $T[X \cup\{v, w\}]$ is decomposable.

This obvious fact does not hold for 3-uniform hypergraphs. Let $H_{5}$ be the 3-uniform hypergraph defined on $V\left(H_{5}\right)=\{0, \ldots, 4\}$ by $E\left(H_{5}\right)=\{012,034\}$. As observed at the beginning of Remark $28, H_{5}[\{0,1,2\}]$ is prime. Set $X=\{0,1,2\}$. Since $E\left(H_{5}[X \cup\{3\}]\right)=\{012\}$, we have $3 \in \operatorname{Dis}_{\left(H_{5}, X\right)}$. Similarly, $4 \in \operatorname{Dis}_{\left(H_{5}, X\right)}$. We verify that $H_{5}[X \cup\{3,4\}]$, which is $H_{5}$, is prime. Let $M$ be a module of $H_{5}$ such that $|M| \geq 2$. We have to show that $M=V\left(H_{5}\right)$. By Fact 29 , we have $M \cap X=\varnothing, M \supseteq X$ or $|M \cap X|=1$. Since $034 \in E\left(H_{5}\right),\{3,4\}$ is not a module of $H$. Thus $M \cap X \neq \varnothing$. For a contradiction, suppose that $|M \cap X|=1$, and denote by $y$ the unique element of $M \cap X$. Since $|M| \geq 2, M \backslash\{y\} \neq \varnothing$. Let $v \in M \backslash\{y\}$. As previously seen, $v \in \operatorname{Dis}_{\left(H_{5}, X\right)}$. Moreover, it follows from the third assertion of Fact 29 that $v \in \operatorname{Twi}_{\left(H_{5}, X\right)}(y)$. Hence, $v \in \operatorname{Dis}_{\left(H_{5}, X\right)} \cap X_{H_{5}}(y)$, which contradicts Fact 14. Therefore $|M \cap X| \neq 1$, and hence $M \supseteq X$. Since 
$034 \in E\left(H_{5}\right)$ and $134 \notin E\left(H_{5}\right), X$ is not a module of $H$. Since $034 \in E\left(H_{5}\right)$, $X \cup\{3\}$ and $X \cup\{4\}$ are not modules of $H$. It follows that $M=V\left(H_{5}\right)$. Consequently, $H_{5}$ is prime.

For a contradiction, suppose that $H_{5}$ admits a realization $T_{5}$. It follows from Theorem 19 that $T_{5}$ is prime. Since $012 \in E\left(H_{5}\right), T_{5}[X]$ is a 3 -cycle, so $T_{5}[X]$ is prime. Since $H_{5}[X \cup\{3\}]$ is decomposable, it follows from Theorem 19 that $T_{5}[X \cup\{3\}]$ is decomposable too. Hence, $3 \notin \operatorname{Ext}_{\left(T_{5}, X\right)}$. Suppose for a contradiction that $3 \in \operatorname{Twi}_{\left(T_{5}, X\right)}(y)$, where $y \in X$. Since $012 \in E\left(H_{5}\right)$, we obtain that 013,023 or 123 belongs to $E\left(H_{5}\right)$, which contradicts $E\left(H_{5}\right)=\{012,034\}$. It follows that $3 \notin \operatorname{Twi}_{\left(T_{5}, X\right)}(y)$. It follows from Lemma 22 that $3 \in \operatorname{Dis}_{\left(T_{5}, X\right)}$. Similarly, $4 \in \operatorname{Dis}_{\left(T_{5}, X\right)}$. As previously seen, $X$ is a module of $T_{5}$, and hence $T_{5}$ is decomposable, a contradiction. Consequently, $H_{5}$ is not realizable.

In Lemma 31, we examine the general case by considering distinct elements $v$ and $w$ of $\operatorname{Dis}_{(H, X)}$, where $H$ is a 3 -uniform hypergraph, and $X \nsubseteq V(H)$ such that $H[X]$ is prime.

Lemma 31. Let $H$ be a 3-uniform hypergraph. Consider $X \mp V(H)$ such that $H[X]$ is prime. Let $v$ and $w$ be distinct elements of $\operatorname{Dis}_{(H, X)}$.

1. If $H[X \cup\{v, w\}]$ is decomposable, then $X$ is a module of $H[X \cup\{v, w\}]$.

2. $H[X \cup\{v, w\}]$ is prime if and only if there exist $y, z \in X$ such that yvw $\epsilon$ $E(H)$ and $z v w \notin E(H)$.

Proof. For the first assertion, consider a nontrivial module $M$ of $H[X \cup\{v, w\}]$. It follows from Facts 14 and 29 that $M \cap X=\varnothing$ or $M \supseteq X$. Therefore, we have $M=\{v, w\}, X, X \cup\{v\}$ or $X \cup\{w\}$. To conclude, we show that if $M=$ $\{v, w\}, X \cup\{v\}$ or $X \cup\{w\}$, then $X$ is a module of $H[X \cup\{v, w\}]$. Hence, suppose that $M=\{v, w\}, X \cup\{v\}$ or $X \cup\{w\}$. It suffices to verify that for $e \in E(H[X \cup\{v, w\}])$, we cannot have $e \cap X \neq \varnothing$ and $e \backslash X \neq \varnothing$. If $|e \cap X|=2$, then $e \backslash X=\{v\}$ or $\{w\}$, which contradicts the fact that $X$ is a module of $H[X \cup\{v\}]$ and $H[X \cup\{w\}]$. Moreover, if there exists $y \in X$ such that $e \cap X=\{y\}$, then $e=y v w$, which is not possible because $M=\{v, w\}, X \cup\{v\}$ or $X \cup\{w\}$.

For the second assertion, suppose that there exist $y, z \in X$ such that yvw $\epsilon$ $E(H)$ and $z v w \notin E(H)$. Thus, $X$ is not a module of $H[X \cup\{v, w\}]$. It follows from the first assertion above that $H[X \cup\{v, w\}]$ is prime. Conversely, suppose that $H[X \cup\{v, w\}]$ is prime. In particular, $X$ is not a module of $H[X \cup\{v, w\}]$. Therefore, there exists $e \in E(H[X \cup\{v, w\}])$ such that

$$
|e \cap X|=2 \text { or }
$$

$$
\left\{\begin{array}{l}
|e \cap X|=1 \\
\text { and } \\
\text { there exists } z \in X \text { such that }(e \backslash X) \cup\{z\} \notin E(H[X \cup\{v, w\}]) .
\end{array}\right.
$$

We cannot have $|e n X|=2$ because $X$ is a module of $H[X \cup\{v\}]$ and $H[X \cup\{w\}]$. Consequently, (3) holds. By denoting by $y$ the unique element of $e n X$, we obtain $e=y v w$ and $(e \backslash X) \cup\{z\}=z v w$. Therefore, $y v w \in E(H)$ and $z v w \notin E(H)$. 
Lemma 32. Let $H$ be a 3-uniform hypergraph. Consider $X \mp V(H)$ such that $H[X]$ is prime. Let $v \in \operatorname{Dis}_{(H, X)}$ and $w \in \operatorname{Twi}_{(H, X)}(y)$, where $y \in X$.

1. $X \cup\{w\}$ is a module of $H[X \cup\{v, w\}]$ if and only if $\{y, w\}$ is a module of $H[X \cup\{v, w\}]$.

2. If $H[X \cup\{v, w\}]$ is decomposable, then $X \cup\{w\}$ and $\{y, w\}$ are modules of $H[X \cup\{v, w\}]$.

3. $H[X \cup\{v, w\}]$ is prime if and only if there exists $z \in X$ such that $z v w \in$ $E(H)$.

Proof. For the first assertion, suppose that $X \cup\{w\}$ is a module of $H[X \cup\{v, w\}]$. We have to show that $\{y, w\}$ is a module of $H[X \cup\{v, w\}]$. Hence, consider $e \epsilon$ $E(H[X \cup\{v, w\}])$ such that $e \cap\{y, w\} \neq \varnothing$ and $e \backslash\{y, w\} \neq \varnothing$. For a contradiction, suppose that $e \cap\{y, w\}=\{y, w\}$. We cannot have $e \backslash\{y, w\} \subseteq X$ because $\{y, w\}$ is a module of $H[X \cup\{w\}]$. Moreover, we cannot have $e \backslash\{y, w\}=\{v\}$ because $X \cup\{w\}$ is a module of $H[X \cup\{v, w\}]$. It follows that $e \cap\{y, w\}=\{y\}$ or $\{w\}$. For a contradiction, suppose that $v \in e$. Denote by $z$ the unique element of $e \cap(X \backslash\{y\})$. We get $e=y z v$ or $z v w$, which is impossible because $X \cup\{w\}$ is a module of $H[X \cup\{v, w\}]$. Consequently, we obtain

$$
\left\{\begin{array}{l}
e \cap\{y, w\}=\{y\} \text { or }\{w\} . \\
\text { and } \\
e \backslash\{y, w\} \subseteq X
\end{array}\right.
$$

Since $\{y, w\}$ is a module of $H[X \cup\{w\}]$, we obtain

$$
(e \backslash(e \cap\{y, w\})) \cup(\{y, w\} \backslash e) \in E(H[X \cup\{v, w\}]) .
$$

Conversely, suppose that $\{y, w\}$ is a module of $H[X \cup\{v, w\}]$. Since $X$ is a module of $H[X \cup\{v\}]$, it follows from Proposition 9 (see Assertion (A5) of Definition 8) that $X \cup\{w\}$ is a module of $H[X \cup\{v, w\}]$.

For the second assertion, consider a nontrivial module $M$ of $H[X \cup\{v, w\}]$. By Fact 14, $v$ and $w$ do not belong to the same block of $p_{(H, X)}$. It follows from Fact 29 that $M \supseteq X$ or $|M \cap X|=1$. We distinguish the following two cases.

- Suppose that $M \supseteq X$. By the second assertion of Fact $29, V(H) \backslash M \subseteq$ $\operatorname{Dis}_{(H, X)}$. Since $w \in \operatorname{Twi}_{(H, X)}(y), w \notin \operatorname{Dis}_{(H, X)}$ by Fact 14 . Hence $w \in M$. Since $M$ is a nontrivial module of $H[X \cup\{v, w\}], M=X \cup\{w\}$.

- Suppose that $|M \cap X|=1$. Denote by $z$ the unique element of $M \cap X$. Since $M$ is a nontrivial module of $H[X \cup\{v, w\}], M \backslash X \neq \varnothing$. By the third assertion of Fact 29, $M \backslash X \subseteq \operatorname{Twi}_{(H, X)}(z)$. Since $v \in \operatorname{Dis}_{(H, X)}$, $v \notin \operatorname{Twi}_{(H, X)}(z)$ by Fact 14 . Thus $v \notin M$. Since $M \backslash X \neq \varnothing$, we obtain $M=\{z, w\}$. Therefore $w \in \operatorname{Twi}_{(H, X)}(y) \cap \operatorname{Twi}_{(H, X)}(z)$. By Fact 14, we have $y=z$, so $M=\{y, w\}$. 
It follows from both cases above that $M=X \cup\{w\}$ or $M=\{y, w\}$. By the first assertion above, $X \cup\{w\}$ and $\{y, w\}$ are modules of $H[X \cup\{v, w\}]$.

For the third assertion, suppose that $H[X \cup\{v, w\}]$ is prime. In particular, $\{y, w\}$ is not a module of $H[X \cup\{v, w\}]$. Thus, there exists $e \in E(H[X \cup\{v, w\}])$ such that

$$
\begin{aligned}
& \qquad|e \cap\{y, w\}|=2 \text { or } \\
& \left\{\begin{array}{l}
|e \cap\{y, w\}|=1 \\
\text { and } \\
(e \backslash\{y, w\}) \cup(\{y, w\} \backslash e) \notin E(H[X \cup\{v, w\}]) .
\end{array}\right.
\end{aligned}
$$

We distinguish the following two cases.

- Suppose that $|e \cap\{y, w\}|=2$. There exists $u \in(X \backslash\{y\}) \cup\{v\}$ such that $e=u y w$. We cannot have $u \in X$ because $\{y, w\}$ is a module of $H[X \cup\{w\}]$. Therefore $u=v$, and hence $e=y v w$.

- Suppose that (4) holds. Since $\{y, w\}$ is a module of $H[X \cup\{w\}]$, it follows from (4) that $v \in e$. Denote by $z^{\prime}$ the unique element of $e \cap(X \backslash\{y\})$. We cannot have $y \in e$ because $X$ is a module of $H[X \cup\{v\}]$. Consequently $w \in e$, and hence $e=z^{\prime} v w$.

In both cases, there exists $z \in X$ such that $z v w \in E(H)$.

Conversely, suppose that there exists $z \in X$ such that $z v w \in E(H)$. Thus, $X \cup\{w\}$ is not a module of $H[X \cup\{v, w\}]$. By the second assertion above, $H[X \cup\{v, w\}]$ is prime.

Lemma 33. Let $H$ be a 3-uniform hypergraph. Consider $X \mp V(H)$ such that $H[X]$ is prime. Let $v$ and $w$ be distinct elements of $\operatorname{Twi}_{(H, X)}(y)$, where $y \in X$.

1. If $H[X \cup\{v, w\}]$ is decomposable, then $\{y, v, w\}$ is a module of $H[X \cup$ $\{v, w\}]$.

2. $H[X \cup\{v, w\}]$ is prime if and only if there exists $z \in X \backslash\{y\}$ such that $z v w \in E(H)$.

Proof. For the first assertion, consider a nontrivial module $M$ of $H[X \cup\{v, w\}]$. It follows from Facts 14 and 29 that $M=\{v, w\},\{y, v\},\{y, w\}$ or $\{y, v, w\}$. Suppose that $M=\{v, w\}$ or $\{y, v\}$. Since $\{y, w\}$ is a module of $H[X \cup\{w\}]$, it follows from Proposition 9 (see Assertion (A5) of Definition 8) that $\{y, v, w\}$ is a module of $H[X \cup\{v, w\}]$. Similarly, if $\{y, w\}$ is a module of $H[X \cup\{v, w\}]$, then $\{y, v, w\}$ is a module of $H[X \cup\{v, w\}]$.

For the second assertion, suppose that $H[X \cup\{v, w\}]$ is prime. In particular, $\{y, v, w\}$ is not a module of $H[X \cup\{v, w\}]$. Hence, there exists $e \in E(H[X \cup$ $\{v, w\}])$ such that

$$
\begin{aligned}
& \qquad|e \cap\{y, v, w\}|=2 \text { or } \\
& \left\{\begin{array}{l}
|e \cap\{y, v, w\}|=1 \\
\text { and } \\
(e \backslash\{y, v, w\}) \cup\{z\} \notin E(H[X \cup\{v, w\}]) \text {, where } z \in\{y, v, w\} \backslash e .
\end{array}\right.
\end{aligned}
$$


Suppose that $|e \cap\{y, v, w\}|=1$. We have $e \backslash\{y, v, w\} \subseteq X \backslash\{y\}$. Since $\{y, v\}$ is a module of $H[X \cup\{v\}]$ and $\{y, w\}$ is a module of $H[X \cup\{w\}]$, it follows from $e \in E(H[X \cup\{v, w\}])$ that $(e \backslash\{y, v, w\}) \cup\{z\} \in E(H[X \cup\{v, w\}])$ for every $z \in\{y, v, w\}$. Thus (5) does not hold. Consequently $|e \cap\{y, v, w\}|=2$. Since $\{y, v\}$ is a module of $H[X \cup\{v\}]$ and $\{y, w\}$ is a module of $H[X \cup\{w\}]$, we get $e \cap\{y, v, w\}=\{v, w\}$. By denoting by $z$ the unique element of $e \backslash\{y, v, w\}$, we obtain $z \in X \backslash\{y\}$ and $e=z v w$. Hence $z v w \in E(H)$.

Conversely, suppose that there exists $z \in X \backslash\{y\}$ such that $z v w \in E(H)$. We obtain that $\{y, v, w\}$ is not a module of $H[X \cup\{v, w\}]$. By the first assertion above, $H[X \cup\{v, w\}]$ is prime.

We use the following notation to state Lemma 35.

Notation 34. Let $H$ be a 3-uniform hypergraph.

$$
\text { For } e, f \in\left(\begin{array}{c}
V(H) \\
3
\end{array}\right), e \equiv_{H} f \text { means }\left\{\begin{array}{l}
e, f \in E(H) \\
\text { or } \\
e, f \notin E(H) .
\end{array}\right.
$$

Lemma 35. Let $H$ be a 3-uniform hypergraph. Consider $X \mp V(H)$ such that $H[X]$ is prime. Let $v \in \operatorname{Twi}_{(H, X)}(y)$ and $w \in \operatorname{Twi}_{(H, X)}(z)$, where $y$ and $z$ are distinct elements of $X$.

1. $\{y, v\}$ is a module of $H[X \cup\{v, w\}]$ if and only if $\{z, w\}$ is a module of $H[X \cup\{v, w\}]$.

2. If $H[X \cup\{v, w\}]$ is decomposable, then $\{y, v\}$ and $\{z, w\}$ are modules of $H[X \cup\{v, w\}]$.

3. $H[X \cup\{v, w\}]$ is prime if and only if

$$
\left\{\begin{array}{l}
E(H) \cap\{y v w, z v w\} \neq \varnothing \\
\text { or } \\
\text { there exists } z^{\prime} \in X \backslash\{y, z\} \text { such that } z^{\prime} y z \neq_{H} z^{\prime} v w .
\end{array}\right.
$$

Proof. The first assertion follows from Proposition 9 (see Assertion (A4) of Definition 8). For the second assertion, consider a nontrivial module $M$ of $H[X \cup\{v, w\}]$. It follows from Facts 14 and 29 that $M=\{y, v\}$ or $\{z, w\}$. By the first assertion above, $\{y, v\}$ and $\{z, w\}$ are modules of $H[X \cup\{v, w\}]$.

For the third assertion, suppose that $H[X \cup\{v, w\}]$ is prime. In particular, $\{y, v\}$ is not a module of $H[X \cup\{v, w\}]$. Hence, there exists $e \in E(H[X \cup\{v, w\}])$ such that

$$
\begin{gathered}
\qquad y, v\} \subseteq e \text { or } \\
\left\{\begin{array}{l}
|e \cap\{y, v\}|=1 \\
\text { and } \\
(e \backslash\{y, v\}) \cup(\{y, v\} \backslash e) \notin E(H[X \cup\{v, w\}]) .
\end{array}\right.
\end{gathered}
$$


Suppose that $\{y, v\} \subseteq e$. Since $\{y, v\}$ is a module of $H[X \cup\{v\}], w \in e$. Hence $e=y v w$. Therefore yvw $\in E(H)$, and hence (6) holds. Now, suppose that (7) holds. Since $\{y, v\}$ is a module of $H[X \cup\{v\}], w \in e$. We distinguish the following two cases.

- Suppose that $y \in e$, hence $v \notin e$. Since $\{z, w\}$ is a module of $H[X \cup\{w\}]$, $z \notin e$. Therefore, there exists $z^{\prime} \in X \backslash\{y, z\}$ such that $z^{\prime} \in e$. We obtain $e=z^{\prime} y w$, so $z^{\prime} y w \in E(H)$. Since $\{z, w\}$ is a module of $H[X \cup\{w\}]$, $z^{\prime} y z \in E(H)$. Furthermore, $(e \backslash\{y\}) \cup\{v\} \notin E(H[X \cup\{v, w\}])$ by (7). Therefore $z^{\prime} v w \notin E(H)$. It follows that (6) holds.

- Suppose that $v \in e$, hence $y \notin e$. If $z \in e$, then $e=z v w$, so $z v w \in E(H)$, and hence (6) holds. Lastly, suppose that $z \notin e$. There exists $z^{\prime} \in X \backslash\{y, z\}$ such that $z^{\prime} \in e$. We get $e=z^{\prime} v w$, and hence $z^{\prime} v w \in E(H)$. Furthermore, $(e \backslash\{v\}) \cup\{y\} \notin E(H[X \cup\{v, w\}])$ by $(7)$. Therefore $z^{\prime} y w \notin E(H)$. Since $\{z, w\}$ is a module of $H[X \cup\{w\}], z^{\prime} y z \notin E(H)$. It follows that (6) holds.

Conversely, suppose that (6) holds. To begin, suppose that $y v w \in E(H)$. It follows that $\{y, v\}$ is not a module of $H[X \cup\{v, w\}]$. By the second assertion above, $H[X \cup\{v, w\}]$ is prime. Similarly, if $z v w \in E(H)$, then $H[X \cup\{v, w\}]$ is prime. Finally, suppose that there exists $z^{\prime} \in X \backslash\{y, z\}$ such that $z^{\prime} v w \neq_{H} z^{\prime} y z$ (see Notation 34). Since $\{y, v\}$ is a module of $H[X \cup\{v\}]$, we get $z^{\prime} y z \equiv_{H} z^{\prime} z v$. Thus, $z^{\prime} z v \not_{H} z^{\prime} v w$. It follows that $\{z, w\}$ is not a module of $H[X \cup\{v, w\}]$. By the second assertion above, $H[X \cup\{v, w\}]$ is prime.

\section{Proof of Theorem 15}

We begin the section with two remarks on Corollary 24.

Remark 36. The analogue of Corollary 24 does not hold for prime hypergraphs. Indeed, consider a hypergraph $H$ such that $E(H)=\{X, V(H)\}$, where $|X| \geq 3$ and $|V(H) \backslash X| \geq 2$. It follows from Remark 28 that $H$ and $H[X]$ are prime. Furthermore, for every nonempty $Y \nsubseteq V(H) \backslash X, X$ is a (nontrivial) module of $H[X \cup Y]$, so $H[X \cup Y]$ is decomposable.

Remark 37. The analogue of Corollary 24 does not hold for prime 3 -uniform hypergraphs. Indeed, given $n \geq 4$, consider the 3-uniform hypergraph $H$ defined on $V(H)=\{0, \ldots, n+3\}$ by

$$
\begin{aligned}
E(H) & =\{i(i+1)(i+2): 0 \leq i \leq n-2\} \\
& \cup\{(n-2)(n-1)(n+1),(n-2)(n-1)(n+2),(n+1)(n+2)(n+3)\} .
\end{aligned}
$$

Proceeding by induction on $p \in\{4, \ldots, n\}$, it is easy to verify that $H[\{0, \ldots, p\}]$ is prime. For

$$
X=\{0, \ldots, n\},
$$

we have $H[X]$ is prime. We have $n+1, n+2 \in \operatorname{Twi}_{(H, X)}(n)$, and $n+3 \in \operatorname{Dis}_{(H, X)}$. Thus, $H[X \cup\{v\}]$ is decomposable for every $v \in V(H) \backslash X$. Moreover, it follows 
from Lemmas 32 and 33 that $H[X \cup\{v, w\}]$ is decomposable for distinct $v, w \epsilon$ $V(H) \backslash X$. Lastly, by using Fact 29 , it is not difficult to verify that $H$ is prime.

Given Remark 36, we conjecture the following.

Conjecture 38. Let $H$ be a prime hypergraph. Consider $X \mp V(H)$ such that $H[X]$ is prime. There exists $Y \subseteq V(H) \backslash X$ such that $H[X \cup Y]$ is prime, and

$$
1 \leq|Y| \leq \max (\{|e|: e \in E(H)\}) .
$$

Theorem 15 provides a positive answer to Conjecture 38 for prime 3-uniform hypergraphs.

Remark 39. As mentioned at the beginning of Section 1, we do not use the classic definition of a module of a hypergraph (see Definition 6). The classic definition leads to a new type of prime hypergraphs. Conjecture 38 can be stated for this new type as well. Of course, an analogue of Lemma 12 has to be found too.

Proof of Theorem 15. We suppose that

$$
\text { for any } v, w \in V(H) \backslash X, H[X \cup\{v, w\}] \text { is decomposable. }
$$

Observe that (8) implies $\operatorname{Ext}_{(H, X)}=\varnothing$.

To begin, suppose that $\operatorname{Dis}_{(H, X)} \neq \varnothing$. Since $H$ is prime, $V(H) \backslash \operatorname{Dis}_{(H, X)}$ is not a module of $H$. Thus, there exists $e \in E(H)$ such that

$$
\left|e \cap \operatorname{Dis}_{(H, X)}\right|=1 \quad \text { or }
$$

$$
\begin{cases}\left|e \cap \operatorname{Dis}_{(H, X)}\right|=2 & \\ \text { and there exists } \beta \in V(H) \backslash \operatorname{Dis}_{(H, X)} & \text { such that } \\ & \left(e \cap \operatorname{Dis}_{(H, X)}\right) \cup\{\beta\} \notin E(H) .\end{cases}
$$

We distinguish the following cases.

1. Suppose that $\left|e \cap \operatorname{Dis}_{(H, X)}\right|=1$. Denote by $u$ the unique element of $e n$ $\operatorname{Dis}_{(H, X)}$. Since $X$ is a module of $H[X \cup\{u\}], e n\left(V(H) \backslash\left(X \cup \operatorname{Dis}_{(H, X)}\right)\right) \neq$ $\varnothing$. Let $v \in e \cap\left(V(H) \backslash\left(X \cup \operatorname{Dis}_{(H, X)}\right)\right)$. Since $\operatorname{Ext}_{(H, X)}=\varnothing$, it follows from Fact 14 that $v \in \operatorname{Twi}_{(H, X)}(y)$, where $y \in X$. If $e \backslash\{u, v\} \subseteq X$, then it follows from the third assertion of Lemma 32 that $H[X \cup\{u, v\}]$ is prime, which contradicts (8). Thus $e \cap X=\varnothing$. Denote by $w$ the unique element of $e \backslash\{u, v\}$. As previously for $v$, we have $w \in \operatorname{Twi}_{(H, X)}(z)$, where $z \in X$. We show that

$$
H[X \cup e] \text { is prime. }
$$

Let $M$ be a module of $H[X \cup e]$ such that $|M| \geq 2$. We have to prove that $M=X \cup e$. It follows from Fact 29 that $X \cup\{v, w\} \subseteq M, M=\{y, v\}$ or $\{z, w\}$, and, when $y=z, M=\{y, v, w\}$ or $\{v, w\}$. Since $v, w \in e,\{v, w\}$, $\{y, v, w\}$ and $X \cup\{v, w\}$ are not modules of $H[X \cup e]$. Moreover, suppose 
for a contradiction that $\{y, v\}$ is a module of $H[X \cup e]$. Since $e=u v w$, we obtain $y u w \in E(H)$. By the third assertion of Lemma $32, H[X \cup\{u, w\}]$ is prime, which contradicts (8). Therefore, $\{y, v\}$ is not a module of $H[X \cup e]$. Similarly, $\{z, w\}$ is not a module of $H[X \cup e]$. Consequently, it follows that $X \cup\{v, w\} \mp M$, so $M=X \cup e$.

2. Suppose that (9) holds. Denote the elements of $e \cap \operatorname{Dis}_{(H, X)}$ by $v$ and $w$. Furthermore, denote by $\alpha$ the unique element of $e \backslash\{v, w\}$. We get $\alpha v w \epsilon$ $E(H)$. By $(9)$, there exists $\beta \in V(H) \backslash \operatorname{Dis}_{(H, X)}$ such that $\beta v w \notin E(H)$. Moreover, $H[X \cup\{v, w\}]$ is decomposable by (8). It follows from the first assertion of Lemma 31 that

$$
\begin{array}{r}
\text { either } y v w \in E(H) \text { for every } y \in X \\
\text { or } y v w \notin E(H) \text { for every } y \in X .
\end{array}
$$

Set

$$
\gamma=\left\{\begin{array}{l}
\alpha \text { if (11) holds } \\
\text { or } \\
\beta \text { if (10) holds }
\end{array}\right.
$$

Since $\gamma=\alpha$ or $\beta, \gamma \notin \operatorname{Dis}_{(H, X)}$. It follows from (12) that

$$
\left\{\begin{array}{l}
\gamma v w \in E(H) \Longleftrightarrow \text { for every } y \in X, y v w \notin E(H), \\
\text { and } \\
\gamma v w \notin E(H) \Longleftrightarrow \text { for every } y \in X, y v w \in E(H) .
\end{array}\right.
$$

Thus

$$
\gamma \in V(H) \backslash\left(X \cup \operatorname{Dis}_{(H, X)}\right)
$$

Since $\operatorname{Ext}_{(H, X)}=\varnothing$, it follows from Fact 14 that there exists $z \in X$ such that $\gamma \in \operatorname{Twi}_{(H, X)}(z)$. We prove that

$$
H[X \cup\{\gamma, v, w\}] \text { is prime. }
$$

Let $M$ be a module of $H[X \cup\{\gamma, v, w\}]$ such that $|M| \geq 2$. We have to show that $M=X \cup\{\gamma, v, w\}$. It follows from Fact 29 that $M=\{v, w\},\{z, \gamma\}$ or $M \supseteq X \cup\{\gamma\}$. It follows from (13) that $\{z, \gamma\}$ and $X \cup\{\gamma\}$ are not modules of $H[X \cup\{\gamma, v, w\}]$. Moreover, it follows from (13) that there exists $\delta \in X \cup\{\gamma\}$ such that $\delta v w \in E(H)$. Therefore, $\{v, w\}, X \cup\{\gamma, v\}$ and $X \cup\{\gamma, w\}$ are not modules of $H[X \cup\{\gamma, v, w\}]$. Consequently $M=$ $X \cup\{\gamma, v, w\}$.

Now, suppose that $\operatorname{Dis}_{(H, X)}=\varnothing$. Since $\operatorname{Ext}_{(H, X)}=\varnothing$, it follows from Fact 14 that there exists $y \in X$ such that $\operatorname{Twi}_{(H, X)}(y) \neq \varnothing$. Hence, $\{y\} \cup \operatorname{Twi}_{(H, X)}(y)$ is not a module of $H$. Therefore, there exists $e \in E(H)$ such that

$$
\left|e \cap\left(\{y\} \cup \operatorname{Twi}_{(H, X)}(y)\right)\right|=2 \text { or }
$$




$$
\left\{\begin{aligned}
\left|e \cap\left(\{y\} \cup \operatorname{Twi}_{(H, X)}(y)\right)\right| & =1 \\
\text { and there exists } \beta \in\{y\} \cup & \operatorname{Twi}_{(H, X)}(y) \text { such that } \\
& \left(e \backslash\left(\{y\} \cup \operatorname{Twi}_{(H, X)}(y)\right)\right) \cup\{\beta\} \notin E(H) .
\end{aligned}\right.
$$

We distinguish the following two cases.

1. Suppose that $\left|e n\left(\{y\} \cup \operatorname{Twi}_{(H, X)}(y)\right)\right|=2$. We have $\left|e n \operatorname{Twi}_{(H, X)}(y)\right|=1$ or 2 . For a contradiction, suppose that $\left|e \cap \operatorname{Twi}_{(H, X)}(y)\right|=1$. Denote by $v$ the unique element of $e \cap \operatorname{Twi}_{(H, X)}(y)$. We get $e \cap\left(\{y\} \cup \operatorname{Twi}_{(H, X)}(y)\right)=\{y, v\}$. Denote by $w$ the unique element of $e \backslash\{y, v\}$. We have $e=y v w$, and hence $y v w \in E(H)$. Since $\{y, v\}$ is a module of $H[X \cup\{v\}], w \notin X$. Thus $\left.w \notin X \cup \operatorname{Twi}_{(H, X)}(y)\right)$. Since $\operatorname{Ext}_{(H, X)}=\varnothing$ and $\operatorname{Dis}_{(H, X)}=\varnothing$, it follows from Fact 14 that there exists $z \in X \backslash\{y\}$ such that $w \in \operatorname{Twi}_{(H, X)}(z)$. Since $y v w \in E(H)$, it follows from the third assertion of Lemma 35 that $H[X \cup$ $\{v, w\}]$ is prime, which contradicts (8). It follows that $\left|e \cap \operatorname{Twi}_{(H, X)}(y)\right|=$ 2. Denote by $u^{\prime}$ and $v^{\prime}$ the elements of $e \cap \operatorname{Twi}_{(H, X)}(y)$, and denote by $w^{\prime}$ the unique element of $e \backslash\left\{u^{\prime}, v^{\prime}\right\}$. We have $e=u^{\prime} v^{\prime} w^{\prime}$, and hence $u^{\prime} v^{\prime} w^{\prime} \in E(H)$. Clearly, $w^{\prime} \notin\{y\} \cup \operatorname{Twi}_{(H, X)}(y)$. If $w^{\prime} \in X$, then it follows from the second assertion of Lemma 33 that $H\left[X \cup\left\{u^{\prime}, v^{\prime}\right\}\right]$ is prime, which contradicts (8). Thus $w^{\prime} \notin X$, so $w^{\prime} \in V(H) \backslash\left(X \cup \operatorname{Twi}_{(H, X)}(y)\right)$. Since $\operatorname{Ext}_{(H, X)}=\varnothing$ and $\operatorname{Dis}_{(H, X)}=\varnothing$, it follows from Fact 14 that there exists $z \in X \backslash\{y\}$ such that $w^{\prime} \in \operatorname{Twi}_{(H, X)}(z)$. We prove that

$$
H\left[X \cup\left\{u^{\prime}, v^{\prime}, w^{\prime}\right\}\right] \text { is prime. }
$$

Let $M$ be a module of $H\left[X \cup\left\{u^{\prime}, v^{\prime}, w^{\prime}\right\}\right]$ such that $|M| \geq 2$. We have to show that $M=X \cup\left\{u^{\prime}, v^{\prime}, w^{\prime}\right\}$. It follows from Fact 29 that $M=$ $\left\{u^{\prime}, v^{\prime}\right\},\left\{y, u^{\prime}, v^{\prime}\right\},\left\{y, u^{\prime}\right\},\left\{y, v^{\prime}\right\},\left\{z, w^{\prime}\right\}$ or $X \cup\left\{u^{\prime}, v^{\prime}, w^{\prime}\right\}$. Since $u^{\prime} v^{\prime} w^{\prime} \in$ $E(H),\left\{u^{\prime}, v^{\prime}\right\}$ and $\left\{y, u^{\prime}, v^{\prime}\right\}$ are not modules of $H\left[X \cup\left\{u^{\prime}, v^{\prime}, w^{\prime}\right\}\right]$. Furthermore, $H\left[X \cup\left\{v^{\prime}, w^{\prime}\right\}\right]$ is decomposable by (8). It follows from the third assertion of Lemma 35 that $y v^{\prime} w^{\prime} \notin E(H)$. Since $u^{\prime} v^{\prime} w^{\prime} \in E(H)$, $\left\{y, u^{\prime}\right\}$ is not a module of $H\left[X \cup\left\{u^{\prime}, v^{\prime}, w^{\prime}\right\}\right]$. Similarly, $\left\{y, v^{\prime}\right\}$ is not a module of $H\left[X \cup\left\{u^{\prime}, v^{\prime}, w^{\prime}\right\}\right]$. Lastly, $H\left[X \cup\left\{u^{\prime}, v^{\prime}\right\}\right]$ is decomposable by (8). It follows from the second assertion of Lemma 33 that $z u^{\prime} v^{\prime} \notin E(H)$. Since $u^{\prime} v^{\prime} w^{\prime} \in E(H),\left\{z, w^{\prime}\right\}$ is not a module of $H\left[X \cup\left\{u^{\prime}, v^{\prime}, w^{\prime}\right\}\right]$. Consequently, we obtain $M=X \cup\left\{u^{\prime}, v^{\prime}, w^{\prime}\right\}$.

2. Suppose that (14) holds. Denote by $\alpha$ the unique element of $e \cap(\{y\} \cup$ $\left.\operatorname{Twi}_{(H, X)}(y)\right)$. Furthermore, denote by $\gamma$ and $\delta$ the elements of $e \backslash(\{y\} \cup$ $\left.\operatorname{Twi}_{(H, X)}(y)\right)$. Hence $e=\alpha \gamma \delta$. By (14), $\alpha \gamma \delta \neq_{H} \beta \gamma \delta$ (see Notation 34). Therefore, there exists $\alpha^{\prime} \in\{\alpha, \beta\}$ such that

$$
\alpha^{\prime} \gamma \delta \neq_{H} y \gamma \delta .
$$

We obtain $\alpha^{\prime} \in \operatorname{Twi}_{(H, X)}(y)$. Since $\left\{y, \alpha^{\prime}\right\}$ is a module of $H\left[X \cup\left\{\alpha^{\prime}\right\}\right]$, it follows from (15) that $\{\gamma, \delta\} \backslash X \neq \varnothing$. For instance, assume that $\gamma \notin X$. Since $\operatorname{Ext}_{(H, X)}=\varnothing$ and $\operatorname{Dis}_{(H, X)}=\varnothing$, it follows from Fact 14 that there 
exists $z \in X$ such that $\gamma \in \operatorname{Twi}_{(H, X)}(z)$. Since $\gamma \notin \operatorname{Twi}_{(H, X)}(y)$, we have $y \neq z$. For a contradiction, suppose that $\delta \in X$. It follows from (15) that

$$
\left\{y, \alpha^{\prime}\right\} \text { is not a module of } H\left[X \cup\left\{\alpha^{\prime}, \gamma\right\}\right] \text {. }
$$

By the second assertion of Lemma 35, $H\left[X \cup\left\{\alpha^{\prime}, \gamma\right\}\right]$ is prime, which contradicts (8). Therefore $\delta \notin X$. Since $\operatorname{Ext}_{(H, X)}=\varnothing$ and $\operatorname{Dis}_{(H, X)}=$ $\varnothing$, it follows from Fact 14 that there exists $z^{\prime} \in X \backslash\{y\}$ such that $\delta \in$ $\operatorname{Twi}_{(H, X)}\left(z^{\prime}\right)$. We prove that

$$
H\left[X \cup\left\{\alpha^{\prime}, \gamma, \delta\right\}\right] \text { is prime. }
$$

Let $M$ be a module of $H\left[X \cup\left\{\alpha^{\prime}, \gamma, \delta\right\}\right]$ such that $|M| \geq 2$. We have to show that $M=X \cup\left\{\alpha^{\prime}, \gamma, \delta\right\}$. It follows from Fact 29 that $M=X \cup$ $\left\{\alpha^{\prime}, \gamma, \delta\right\},\left\{y, \alpha^{\prime}\right\},\{z, \gamma\}$ or $\left\{z^{\prime}, \delta\right\}$, and, when $z=z^{\prime}, M=\{\gamma, \delta\}$ or $\{z, \gamma, \delta\}$. By (15), $y \gamma \delta \in E(H)$ or $\alpha^{\prime} \gamma \delta \in E(H)$. Thus, $\{\gamma, \delta\}$ and $\{z, \gamma, \delta\}$ are not modules of $H\left[X \cup\left\{\alpha^{\prime}, \gamma, \delta\right\}\right]$. Moreover, it follows from (16) and Proposition 9 (see Assertion (A2) of Definition 8) that

$$
\left\{y, \alpha^{\prime}\right\} \text { is not a module of } H\left[X \cup\left\{\alpha^{\prime}, \gamma, \delta\right\}\right] \text {. }
$$

Finally, suppose for a contradiction that $\{z, \gamma\}$ is a module of $H[X \cup$ $\left.\left\{\alpha^{\prime}, \gamma, \delta\right\}\right]$. By (8), $H\left[X \cup\left\{\alpha^{\prime}, \delta\right\}\right]$ is decomposable. By the second assertion of Lemma 35, $\left\{y, \alpha^{\prime}\right\}$ is a module of $H\left[X \cup\left\{\alpha^{\prime}, \delta\right\}\right]$. It follows from Proposition 9 (see Assertion (A4) of Definition 8) that $\left\{y, \alpha^{\prime}\right\}$ is a module of $H\left[X \cup\left\{\alpha^{\prime}, \gamma, \delta\right\}\right]$, which contradicts (17). Consequently, $\{z, \gamma\}$ is not a module of $H\left[X \cup\left\{\alpha^{\prime}, \gamma, \delta\right\}\right]$. Similarly, $\left\{z^{\prime}, \delta\right\}$ is not a module of $H\left[X \cup\left\{\alpha^{\prime}, \gamma, \delta\right\}\right]$. It follows that $M=X \cup\left\{\alpha^{\prime}, \gamma, \delta\right\}$.

Proof of Corollary 16. Consider a prime 3-uniform hypergraph $H$ such that $|V(H)| \geq 4$. By Lemma 12 , there exists $X \subseteq V(H)$ such that $|X|=3$ and $H[X]$ is prime. Hence, $|X|<|V(H)|$. Consider a maximal proper subset $Z$ of $V(H)$ such that $H[Z]$ is prime. For a contradiction, suppose that $|V(H) \backslash Z| \geq 4$. By Theorem 15, there exists $Z^{\prime} \subseteq V(H) \backslash Z$ such that $1 \leq\left|Z^{\prime}\right| \leq 3$ and $H\left[Z \cup Z^{\prime}\right]$ is prime. Since $|V(H) \backslash Z| \geq 4$ and $1 \leq\left|Z^{\prime}\right| \leq 3$, we obtain $Z \cup Z^{\prime} \nsubseteq V(H)$, which contradicts the maximality of $Z$. Consequently, we have $|V(H) \backslash Z| \leq 3$. By setting $Y=V(H) \backslash Z$, we obtain $1 \leq|Y| \leq 3$ and $H-Y$ is prime.

\section{Proof of Theorem 17}

We use the next lemma to establish Theorem 17.

Lemma 40. Let $H$ be a 3-uniform hypergraph. Consider $X \mp V(H)$ such that $H[X]$ is prime. Let $v \in \operatorname{Twi}_{(H, X)}(y)$, where $y \in X$. Set

$$
Y=(X \backslash\{y\}) \cup\{v\} .
$$

We have $H[Y]$ is prime. Moreover, the following three assertions hold. 
1. For every $w \in \operatorname{Twi}_{(H, X)}(y) \backslash\{v\}$, if $H[X \cup\{v, w\}]$ is decomposable, then $w \in \operatorname{Twi}_{(H, Y)}(v)$.

2. Given $z \in X \backslash\{y\}$ and $w \in \operatorname{Twi}_{(H, X)}(z)$, if $H[X \cup\{v, w\}]$ is decomposable, then $w \in \operatorname{Twi}_{(H, Y)}(z)$.

3. For every $w \in \operatorname{Dis}_{(H, X)}$, if $H[X \cup\{v, w\}]$ is decomposable, then $w \in$ $\operatorname{Dis}_{(H, X)}$.

Proof. Since $\{y, v\}$ is a module of $H[X \cup\{v\}]$, the function

$$
\begin{aligned}
X & \longrightarrow Y \\
y & \longmapsto v \\
z \in X \backslash\{y\} & \longmapsto z,
\end{aligned}
$$

is an isomorphism from $H[X]$ onto $H[Y]$. Hence $H[Y]$ is prime.

Let $w \in \operatorname{Twi}_{(H, X)}(y) \backslash\{v\}$ be such that $H[X \cup\{v, w\}]$ is decomposable. By the first assertion of Lemma 33, \{y,v,w\} is a module of $H[X \cup\{v, w\}]$. By Proposition 9 (see Assertion (A2) of Definition 8), $\{v, w\}$ is a module of $H[(X \cup\{v, w\}) \backslash\{y\}]$, that is, $H[Y \cup\{w\}]$. It follows that $w \in \operatorname{Twi}_{(Y, X)}(v)$.

Given $z \in X \backslash\{y\}$, consider $w \in \operatorname{Twi}_{(H, X)}(z)$ such that $H[X \cup\{v, w\}]$ is decomposable. By the second assertion of Lemma 35, $\{z, w\}$ is a module of $H[X \cup\{v, w\}]$. By Proposition 9 (see Assertion (A2) of Definition 8), $\{z, w\}$ is a module of $H[Y \cup\{w\}]$. It follows that $w \in \operatorname{Twi}_{(H, Y)}(z)$.

Let $w \in \operatorname{Dis}_{(H, X)}$ such that $H[X \cup\{v, w\}]$ is decomposable. By the second assertion of Lemma 32, $X \cup\{v\}$ is a module of $H[X \cup\{v, w\}]$. By Proposition 9 (see Assertion (A2) of Definition 8), $Y$ is a module of $H[Y \cup\{w\}]$. It follows that $w \in \operatorname{Dis}_{(H, Y)}$.

Proof of Theorem 17. We suppose that

$$
H-\{v, w\} \text { is decomposable for any } v, w \in(V(H) \backslash X) \cup \underline{X},
$$

where $v=w$ is allowed. Consequently, we have to prove that $H$ is decomposable.

Using Theorem 15 several times, beginning with $H[X]$, we obtain $Y \subseteq V(H)$ such that $X \subseteq Y, H[Y]$ is prime, and $1 \leq|V(H) \backslash Y| \leq 3$. Set

$$
\underline{Y}=\left\{z \in Y: \operatorname{Twi}_{(H, Y)}(z) \neq \varnothing\right\} .
$$

We prove that

$$
(V(H) \backslash Y) \cup \underline{Y} \subseteq(V(H) \backslash X) \cup \underline{X} .
$$

Clearly, $(V(H) \backslash Y) \subseteq(V(H) \backslash X)$. Now, consider $z \in \underline{Y}$. We have $z \in V(H) \backslash X$ or $z \in X$. Hence, suppose that $z \in X$. Since $z \in \underline{Y}$, there exists $v \in V(H) \backslash Y$ such that $\{z, v\}$ is a module of $H[Y \cup\{v\}]$. Since $z \in X$, it follows from Proposition 9 (see Assertion (A2) of Definition 8) that $\{z, v\}$ is a module of $H[X \cup\{v\}]$. Thus $v \in \operatorname{Twi}_{(H, X)}(z)$, so $z \in \underline{X}$. Hence (19) holds. It follows from (18) that

$$
H-\{v, w\} \text { is decomposable for any } v, w \in(V(H) \backslash Y) \cup \underline{Y} .
$$


Furthermore, since $1 \leq|V(H) \backslash Y| \leq 3$, it follows from (20) that $|V(H) \backslash Y|=3$. Denote by $v_{1}, v_{2}$ and $v_{3}$ the elements of $V(H) \backslash Y$. By $(20), \operatorname{Ext}_{(H, Y)}=\varnothing$. Therefore, one of the following seven cases holds. In each of them, we obtain a nontrivial module of $H$.

1. Suppose that $v_{1}, v_{2}, v_{3} \in \operatorname{Dis}_{(H, Y)}$. We show that $Y$ is a module of $H$. Let $e \in E(H)$ such that $e \cap Y \neq \varnothing$ and $e \backslash Y \neq \varnothing$. Since $Y$ is a module of $H\left[Y \cup\left\{v_{i}\right\}\right]$ for $i \in\{1,2,3\}$, there exist distinct $i, j \in\{1,2,3\}$ such that $e \backslash Y=\left\{v_{i}, v_{j}\right\}$. By (20), $H\left[Y \cup\left\{v_{i}, v_{j}\right\}\right]$ is decomposable. It follows from the first assertion of Lemma 31 that $Y$ is a module of $H\left[Y \cup\left\{v_{i}, v_{j}\right\}\right]$. Since $Y$ is a module of $H\left[Y \cup\left\{v_{i}, v_{j}\right\}\right]$, there exists $y \in Y$ such that $e \cap Y=\{y\}$. Furthermore, for every $z \in Y$, we have $(e \backslash\{y\}) \cup\{z\} \in E(H)$. It follows that $Y$ is a module of $H$.

2. Suppose that $v_{1}, v_{2} \in \operatorname{Dis}_{(H, Y)}$ and $v_{3} \in \operatorname{Twi}_{(H, Y)}(y)$, where $y \in Y$. We show that $\left\{y, v_{3}\right\}$ is a module of $H$. Let $e \in E(H)$ such that $e \cap\left\{y, v_{3}\right\} \neq \varnothing$ and $e \backslash\left\{y, v_{3}\right\} \neq \varnothing$.

Suppose that there exists $i \in\{1,2\}$ such that $e \backslash\left\{y, v_{3}\right\} \subseteq Y \cup\left\{v_{i}\right\}$. We get $e \subseteq Y \cup\left\{v_{i}, v_{3}\right\}$. By (20), $H\left[Y \cup\left\{v_{i}, v_{3}\right\}\right]$ is decomposable. By the second assertion of Lemma 32, $\left\{y, v_{3}\right\}$ is a module of $H\left[Y \cup\left\{v_{i}, v_{3}\right\}\right]$. Therefore, there exists $\alpha \in\left\{y, v_{3}\right\}$ such that $e \cap\left\{y, v_{3}\right\}=\{\alpha\}$. Furthermore, $(e \backslash\{\alpha\}) \cup\{\beta\} \in E(H)$, where $\beta$ denotes the unique element of $\left\{y, v_{3}\right\} \backslash\{\alpha\}$.

Now, suppose that $e \backslash\left\{y, v_{3}\right\} \nsubseteq Y \cup\left\{v_{1}\right\}$ and $e \backslash\left\{y, v_{3}\right\} \nsubseteq Y \cup\left\{v_{2}\right\}$. Since $e \backslash\left\{y, v_{3}\right\} \nsubseteq Y \cup\left\{v_{1}\right\}$, we have $v_{2} \in e$. Similarly, $v_{1} \in e$. Thus $e \backslash\left\{y, v_{3}\right\}=$ $\left\{v_{1}, v_{2}\right\}$. We have to verify that

$$
\left.v_{1} v_{2} v_{3} \equiv_{H} v_{1} v_{2} y \quad \text { (see Notation } 34\right) .
$$

Let $z \in Y \backslash\{y\}$. By (20), $H\left[Y \cup\left\{v_{1}, v_{2}\right\}\right]$ is decomposable. By the first assertion of Lemma 31, $Y$ is a module of $H\left[Y \cup\left\{v_{1}, v_{2}\right\}\right]$. Thus

$$
v_{1} v_{2} y \equiv_{H} v_{1} v_{2} z \text {. }
$$

Set $Z=(Y \backslash\{y\}) \cup\left\{v_{3}\right\}$. By Lemma $40, H[Z]$ is prime. Clearly, $y \in$ $\operatorname{Twi}_{(H, Z)}\left(v_{3}\right)$. Furthermore, $H\left[Y \cup\left\{v_{1}, v_{3}\right\}\right]$ is decomposable by (20). It follows from the third assertion of Lemma 40 that $v_{1} \in \operatorname{Dis}_{(H, Z)}$. Similarly, $v_{2} \in \operatorname{Dis}_{(H, Z)}$. Since $v_{3} \in \operatorname{Twi}_{(H, Y)}(y), y \in \underline{Y}$. By $(20), H-y$, which is $H\left[Z \cup\left\{v_{1}, v_{2}\right\}\right]$, is decomposable. By the first assertion of Lemma 31, $Z$ is a module of $H\left[Z \cup\left\{v_{1}, v_{2}\right\}\right]$. Therefore

$$
v_{1} v_{2} z \equiv_{H} v_{1} v_{2} v_{3} .
$$

It follows from (22) and (23) that (21) holds.

3. Suppose that $v_{1} \in \operatorname{Dis}_{(H, Y)}$ and $v_{2}, v_{3} \in \operatorname{Twi}_{(H, Y)}(y)$, where $y \in Y$. We prove that $Y \cup\left\{v_{2}, v_{3}\right\}$ is a module of $H$. It suffices to prove that for each $e \in E(H), v_{1} \notin e$. Suppose to the contrary that there exists $e \in E(H)$ such that $v_{1} \in e$. We verify that

$$
e=v_{1} v_{2} v_{3}
$$


Suppose that $v_{3} \notin e$. We obtain $e \in E\left(H\left[Y \cup\left\{v_{1}, v_{2}\right\}\right]\right)$ and $\left|e n\left(Y \cup\left\{v_{2}\right\}\right)\right|=$ 2. Therefore, $Y \cup\left\{v_{2}\right\}$ is not a module of $H\left[Y \cup\left\{v_{1}, v_{2}\right\}\right]$. It follows from the second assertion of Lemma 32 that $H\left[Y \cup\left\{v_{1}, v_{2}\right\}\right]$ is prime, which contradicts (20). Consequently, $v_{3} \in e$. Similarly, $v_{2} \in e$. Hence, (24) holds. Set $Z=(Y \backslash\{y\}) \cup\left\{v_{3}\right\}$. By Lemma $40, H[Z]$ is prime. Clearly, $y \in \operatorname{Twi}_{(H, Z)}\left(v_{3}\right)$. By $(20), H\left[Y \cup\left\{v_{1}, v_{3}\right\}\right]$ is decomposable. It follows from the third assertion of Lemma 40 that $v_{1} \in \operatorname{Dis}_{(H, Z)}$. Furthermore, $H\left[Y \cup\left\{v_{2}, v_{3}\right\}\right]$ is decomposable by (20). It follows from the first assertion of Lemma 40 that $v_{2} \in \operatorname{Twi}_{(H, Z)}\left(v_{3}\right)$. Since $v_{1} \in e \backslash\left(Z \cup\left\{v_{2}\right\}\right)$ and $v_{2}, v_{3} \in$ en $\left(Z \cup\left\{v_{2}\right\}\right), Z \cup\left\{v_{2}\right\}$ is not a module of $H\left[Z \cup\left\{v_{1}, v_{2}\right\}\right]$. It follows from the second assertion of Lemma 32 that $H\left[Z \cup\left\{v_{1}, v_{2}\right\}\right]$ is prime. Clearly, $H\left[Z \cup\left\{v_{1}, v_{2}\right\}\right]=H-y$. Since $v_{2} \in \operatorname{Twi}_{(H, Y)}(y)$, we have $y \in \underline{Y}$, which contradicts (20).

4. Suppose that $v_{1} \in \operatorname{Dis}_{(H, Y)}$ and $v_{2} \in \operatorname{Twi}_{(H, Y)}(y)$ and $v_{3} \in \operatorname{Twi}_{(H, Y)}(z)$, where $y$ and $z$ are distinct elements of $Y$. We prove that $Y \cup\left\{v_{2}, v_{3}\right\}$ is a module of $H$. It suffices to prove that

$$
\text { for each } e \in E(H), v_{1} \notin e \text {. }
$$

Since $v_{1} \in \operatorname{Dis}_{(H, Y)}$, we have

$$
y^{\prime} z^{\prime} v_{1} \notin E(H) \text { for distinct elements } y^{\prime} \text { and } z^{\prime} \text { of } Y \text {. }
$$

By (20), $H\left[Y \cup\left\{v_{1}, v_{2}\right\}\right]$ is decomposable. By the second assertion of Lemma 32, $\left\{y, v_{2}\right\}$ is a module of $H\left[Y \cup\left\{v_{1}, v_{2}\right\}\right]$. Hence $y v_{1} v_{2} \notin E(H)$. Let $t \in Y \backslash\{y\}$. By $(26), t y v_{1} \notin E(H)$. Since $\left\{y, v_{2}\right\}$ is a module of $H\left[Y \cup\left\{v_{1}, v_{2}\right\}\right]$, we obtain $t v_{1} v_{2} \notin E(H)$. Therefore, $y^{\prime} v_{1} v_{2} \notin E(H)$ for every $y^{\prime} \in Y$. Similarly, we have $z^{\prime} v_{1} v_{3} \notin E(H)$ for every $z^{\prime} \in Y$.

Lastly, we verify that $v_{1} v_{2} v_{3} \notin E(H)$. Set $Z=(Y \backslash\{y\}) \cup\left\{v_{2}\right\}$. By Lemma $40, H[Z]$ is prime. Clearly, $y \in \operatorname{Twi}_{(H, Z)}\left(v_{2}\right)$. Furthermore, it follows from (20) and Lemma 40 that $v_{1} \in \operatorname{Dis}_{(H, Z)}$ and $v_{3} \in \operatorname{Twi}_{(H, Z)}(z)$. Moreover, $y \in \underline{Y}$ because $v_{2} \in \operatorname{Twi}_{(H, Y)}(y)$. By (20), $H-y$, which is $H\left[Z \cup\left\{v_{1}, v_{3}\right\}\right]$, is decomposable. By the second assertion of Lemma 32, $Z \cup\left\{v_{3}\right\}$ is a module of $H\left[Z \cup\left\{v_{1}, v_{3}\right\}\right]$. Therefore $v_{1} v_{2} v_{3} \notin E(H)$.

5. Suppose that $v_{1}, v_{2}, v_{3} \in \operatorname{Twi}_{(H, Y)}(y)$, where $y \in Y$. We prove that $\left\{y, v_{1}, v_{2}, v_{3}\right\}$ is a module of $H$. Consider $e \in E(H)$ such that $e \cap\left\{y, v_{1}, v_{2}\right.$, $\left.v_{3}\right\} \neq \varnothing$ and $e \backslash\left\{y, v_{1}, v_{2}, v_{3}\right\} \neq \varnothing$. There exist distinct $i, j \in\{1,2,3\}$ such that $e \cap\left\{y, v_{1}, v_{2}, v_{3}\right\} \subseteq\left\{y, v_{i}, v_{j}\right\}$. Hence $e \subseteq Y \cup\left\{v_{i}, v_{j}\right\}$. By (20), $H\left[Y \cup\left\{v_{i}, v_{j}\right\}\right]$ is decomposable. By the first assertion of Lemma 33, $\left\{y, v_{i}, v_{j}\right\}$ is a module of $H\left[Y \cup\left\{v_{i}, v_{j}\right\}\right]$. It follows that there exists $\alpha \epsilon$ $\left\{y, v_{i}, v_{j}\right\}$ such that $e \cap\left\{y, v_{i}, v_{j}\right\}=\{\alpha\}$. Since $e n\left\{y, v_{1}, v_{2}, v_{3}\right\} \subseteq\left\{y, v_{i}, v_{j}\right\}$, we get

$$
e \cap\left\{y, v_{1}, v_{2}, v_{3}\right\}=\{\alpha\} .
$$

Furthermore, since $\left\{y, v_{i}, v_{j}\right\}$ is a module of $H\left[Y \cup\left\{v_{i}, v_{j}\right\}\right]$, we obtain that

$$
(e \backslash\{\alpha\}) \cup\{\beta\} \in E(H) \text { for every } \beta \in\left\{y, v_{i}, v_{j}\right\} .
$$


It remains to verify that $(e \backslash\{\alpha\}) \cup\left\{v_{k}\right\} \in E(H)$, where $k$ denotes the unique element of $\{1,2,3\} \backslash\{i, j\}$. By $(27)$, we have $(e \backslash\{\alpha\}) \cup\{y\} \in E(H)$. Since $v_{k} \in \operatorname{Twi}_{(H, Y)}(y)$, that is, $\left\{y, v_{k}\right\}$ is a module of $H\left[Y \cup\left\{v_{k}\right\}\right]$, we get $(e \backslash\{\alpha\}) \cup\left\{v_{k}\right\} \in E(H)$.

6. Suppose that $v_{1}, v_{2} \in \operatorname{Twi}_{(H, Y)}(y)$ and $v_{3} \in \operatorname{Twi}_{(H, Y)}(z)$, where $y$ and $z$ are distinct elements of $Y$. We prove that $\left\{z, v_{3}\right\}$ is a module of $H$. Consider $e \in E(H)$ such that $e \cap\left\{z, v_{3}\right\} \neq \varnothing$ and $e \backslash\left\{z, v_{3}\right\} \neq \varnothing$.

Suppose that there exists $i \in\{1,2\}$ such that $e \backslash\left\{z, v_{3}\right\} \subseteq Y \cup\left\{v_{i}\right\}$. We get $e \subseteq Y \cup\left\{v_{i}, v_{3}\right\}$. By (20), $H\left[Y \cup\left\{v_{i}, v_{3}\right\}\right]$ is decomposable. By the second assertion of Lemma 35, \{z, $\left.v_{3}\right\}$ is a module of $H\left[Y \cup\left\{v_{i}, v_{3}\right\}\right]$. Therefore, there exists $\alpha \in\left\{z, v_{3}\right\}$ such that $e \cap\left\{z, v_{3}\right\}=\{\alpha\}$, and $(e \backslash\{\alpha\}) \cup\{\beta\} \in$ $E(H)$, where $\beta$ denotes the unique element of $\left\{z, v_{3}\right\} \backslash\{\alpha\}$.

Now, suppose that $e \backslash\left\{z, v_{3}\right\} \nsubseteq Y \cup\left\{v_{1}\right\}$ and $e \backslash\left\{z, v_{3}\right\} \nsubseteq Y \cup\left\{v_{2}\right\}$. Since $e \backslash\left\{z, v_{3}\right\} \nsubseteq Y \cup\left\{v_{1}\right\}, v_{2} \in e$. Similarly, $v_{1} \in e$. To conclude, we have to verify that $v_{1} v_{2} v_{3} \equiv_{H} z v_{1} v_{2}$. In fact, we have $v_{1} v_{2} v_{3} \notin E(H)$ and $z v_{1} v_{2} \notin E(H)$. Indeed, $H\left[Y \cup\left\{v_{1}, v_{2}\right\}\right]$ is decomposable by $(20)$. By the first assertion of Lemma 33, $\left\{y, v_{1}, v_{2}\right\}$ is a module of $H\left[Y \cup\left\{v_{1}, v_{2}\right\}\right]$. Thus $z v_{1} v_{2} \notin E(H)$. Lastly, set $Z=(Y \backslash\{z\}) \cup\left\{v_{3}\right\}$. By Lemma 40,H[Z] is prime. Clearly, $z \in \operatorname{Twi}_{(H, Z)}\left(v_{3}\right)$. Furthermore, it follows from (20) and Lemma 40 that $v_{1}, v_{2} \in \operatorname{Twi}_{(H, Z)}(y)$. Moreover, $z \in \underline{Y}$ because $v_{3} \in \operatorname{Twi}_{(H, Y)}(z)$. By $(20)$, $H-z$, which is $H\left[Z \cup\left\{v_{1}, v_{2}\right\}\right]$, is decomposable. By the first assertion of Lemma 33, $\left\{y, v_{1}, v_{2}\right\}$ is a module of $H\left[Z \cup\left\{v_{1}, v_{2}\right\}\right]$. Therefore $v_{1} v_{2} v_{3} \notin$ $E(H)$.

7. Suppose that $v_{1} \in \operatorname{Twi}_{(H, Y)}\left(y_{1}\right), v_{2} \in \operatorname{Twi}_{(H, Y)}\left(y_{2}\right)$ and $v_{3} \in \operatorname{Twi}_{(H, Y)}\left(y_{3}\right)$, where $y_{1}, y_{2}$ and $y_{3}$ are distinct elements of $Y$. We prove that $\left\{y_{1}, v_{1}\right\}$ is a module of $H$. Consider $e \in E(H)$ such that $e \cap\left\{y_{1}, v_{1}\right\} \neq \varnothing$ and $e \backslash\left\{y_{1}, v_{1}\right\} \neq \varnothing$.

Suppose that there exists $i \in\{2,3\}$ such that $e \backslash\left\{y_{1}, v_{1}\right\} \subseteq Y \cup\left\{v_{i}\right\}$. We get $e \subseteq Y \cup\left\{v_{1}, v_{i}\right\}$. By (20), $H\left[Y \cup\left\{v_{1}, v_{i}\right\}\right]$ is decomposable. By the second

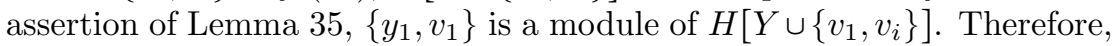
there exists $\alpha \in\left\{y_{1}, v_{1}\right\}$ such that $e \cap\left\{y_{1}, v_{1}\right\}=\{\alpha\}$, and $(e \backslash\{\alpha\}) \cup\{\beta\} \in$ $E(H)$, where $\beta$ denotes the unique element of $\left\{y_{1}, v_{1}\right\} \backslash\{\alpha\}$.

Now, suppose that $e \backslash\left\{y_{1}, v_{1}\right\} \nsubseteq Y \cup\left\{v_{2}\right\}$ and $e \backslash\left\{y_{1}, v_{1}\right\} \nsubseteq Y \cup\left\{v_{3}\right\}$. Since $e \backslash\left\{y_{1}, v_{1}\right\} \nsubseteq Y \cup\left\{v_{2}\right\}, v_{3} \in e$. Similarly, $v_{2} \in e$. To conclude, we have to verify that

$$
v_{1} v_{2} v_{3} \equiv_{H} y_{1} v_{2} v_{3} .
$$

By (20), $H\left[Y \cup\left\{v_{2}, v_{3}\right\}\right]$ is decomposable. By the second assertion of Lemma 35, $\left\{y_{2}, v_{2}\right\}$ and $\left\{y_{3}, v_{3}\right\}$ are modules of $H\left[Y \cup\left\{v_{2}, v_{3}\right\}\right]$. It follows that $y_{1} v_{2} v_{3} \equiv_{H} y_{1} y_{2} y_{3}$. Since $v_{1} \in \operatorname{Twi}_{(H, Y)}\left(y_{1}\right)$, we have $y_{1} y_{2} y_{3} \equiv_{H}$ $y_{2} y_{3} v_{1}$. Therefore,

$$
y_{1} v_{2} v_{3} \equiv_{H} y_{2} y_{3} v_{1} .
$$

Set $Z=\left(Y \backslash\left\{y_{1}\right\}\right) \cup\left\{v_{1}\right\}$. By Lemma 40,H[Z] is prime. Clearly $y_{1} \in$ $\operatorname{Twi}_{(H, Z)}\left(v_{1}\right)$. Furthermore, it follows from $(20)$ and Lemma 40 that $v_{2} \epsilon$ 
$\operatorname{Twi}_{(H, Z)}\left(y_{2}\right)$ and $v_{3} \in \operatorname{Twi}_{(H, Z)}\left(y_{3}\right)$. Moreover, $y_{1} \in \underline{Y}$ because $v_{1} \epsilon$ $\operatorname{Twi}_{(H, Y)}\left(y_{1}\right)$. By $(20), H-y_{1}$, which is $H\left[Z \cup\left\{v_{2}, v_{3}\right\}\right]$, is decomposable. By the second assertion of Lemma 35, $\left\{y_{2}, v_{2}\right\}$ and $\left\{y_{3}, v_{3}\right\}$ are modules of $H\left[Z \cup\left\{v_{2}, v_{3}\right\}\right]$. Consequently, $y_{2} y_{3} v_{1} \equiv_{H} v_{1} v_{2} v_{3}$. It follows that $y_{1} v_{2} v_{3} \equiv_{H}$ $v_{1} v_{2} v_{3}$.

Lastly, a remark on Corollary 18 follows.

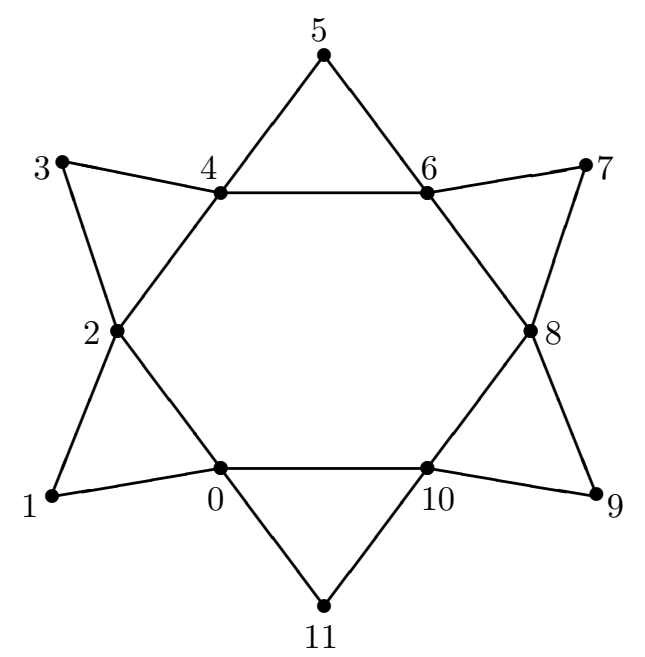

Figure 1: The 3-uniform hypergraph $C_{12}$.

Remark 41. Given $n \geq 3$, the 3 -uniform hypergraph $C_{2 n}$ is defined on $\{0, \ldots, 2 n-$ 1\} by

$E\left(C_{2 n}\right)=\{(2 i)(2 i+1)(2 i+2): 0 \leq i \leq n-2\} \cup\{0(2 n-2)(2 n-1)\}$ (see Figure 1).

The 3-uniform hypergraph $C_{2 n}$ is prime. However, for any distinct $v, w \in$ $\{0, \ldots, 2 n-1\}, C_{2 n}-\{v, w\}$ is disconnected, and hence decomposable (see Remark 11). It follows from Theorems 19 and 26 that $C_{2 n}$ is not realizable. By Corollary 18 , there exists $v \in\{0, \ldots, 2 n-1\}$ such that $C_{2 n}-v$ is prime. Indeed, $C_{2 n}-(2 p+1)$ is prime for $p=0, \ldots, n-1$.

\section{Acknowledgements}

The authors thank both referees for their constructive suggestions that allow for notable improvements to the manuscript. 


\section{Declarations}

Funding: not applicable.

Conflicts of interest/Competing interests: not applicable.

Availability of data and material (data transparency): not applicable.

Code availability (software application or custom code): not applicable.

\section{References}

[1] P. Bonizzoni, G. Della Vedova, An algorithm for the modular decomposition of hypergraphs, J. Algorithms 32 (1999) 65-86.

[2] Y. Boudabbous, P. Ille, B. Jouve, A. Salhi, Critically twin primitive 2structures, Graphs Combin. 31 (2015) 1223-1247.

[3] A. Boussaïri, B. Chergui, P. Ille, M. Zaidi, 3-uniform hypergraphs: modular decomposition and realization by tournaments, Contrib. Discrete Math. 15 (2020) 121-153.

[4] A. Boussaïri, P. Ille, G. Lopez, S. Thomassé, The $C_{3}$-structure of the tournaments, Discrete Math. 277 (2004) 29-43.

[5] M. Burley, J.P. Uhry, Parity graphs, Ann. Discrete Math. 16 (1982) 1-26.

[6] M. Chein, M. Habib, M.C. Maurer, Partitive hypergraphs, Discrete Math. 37 (1981) 35-50.

[7] A. Ehrenfeucht, T. Harju, G. Rozenberg, The Theory of 2-Structures, A Framework for Decomposition and Transformation of Graphs, World Scientific, Singapore, 1999.

[8] A. Ehrenfeucht, G. Rozenberg, Primitivity is hereditary for 2-structures, Theoret. Comput. Sci. 70 (1990) 343-358.

[9] D. Haglin, M. Wolf, On convex subsets in tournaments, SIAM J. Discrete Math. 9 (1996) 63-70.

[10] P. Ille, Indecomposable graphs, Discrete Math. 173 (1997) 71-78.

[11] M. Y. Sayar, Partially critical indecomposable tournaments and partially critical supports, Contrib. Discrete Math. 6 (2011) 52-76.

[12] J.H. Schmerl, W.T. Trotter, Critically indecomposable partially ordered sets, graphs, tournaments and other binary relational structures, Discrete Math. 113 (1993), 191-205.

[13] J. Spinrad, P4-trees and substitution decomposition, Discrete Appl. Math. 39 (1992) 263-291. 